\title{
A Tethering Complex Drives the Terminal Stage of SNARE- Dependent Membrane Fusion
}

Massimo D'Agostino ${ }^{1}$, Herre Jelger Risselada², Anna Lürick ${ }^{3}$, Christian Ungermann ${ }^{3}$, and Andreas Mayer ${ }^{1}$

\author{
${ }^{1}$ Département de Biochimie, Université de Lausanne \\ Chemin des Boveresses 155, CH-1066 Epalinges \\ ${ }^{2}$ Georg-August University, Department of Theoretical Physics, Göttingen \\ Friedrich-Hund-Platz 1, D-37077 Göttingen \\ ${ }^{3}$ University of Osnabrück, Department of Biology/Chemistry Biochemistry section, \\ Barbarastrasse 13, D-49076 Osnabrück
}

Correspondence: Andreas.Mayer@unil.ch

Membrane fusion in eukaryotic cells mediates the biogenesis of organelles, vesicular traffic between them, and exo- and endocytosis of important signaling molecules, such as hormones and neurotransmitters. Distinct tasks in intracellular membrane fusion have been assigned to conserved protein systems. Whereas tether proteins mediate initial recognition and attachment of membranes, SNARE protein complexes are considered as the core fusion engine. They provide mechanical energy to distort membranes and drive them through a hemifusion intermediate towards the formation of a fusion pore $^{1-3}$. This last step is highly energy-demanding ${ }^{4,5}$. We combined the in vivo and in vitro fusion of yeast vacuoles with molecular simulations to show that tether proteins are critical to overcome the final energy barrier to fusion pore formation. SNAREs alone drive vacuoles only into hemifusion. Tether proteins greatly increase the volume of SNARE complexes and deform the site of hemifusion, which lowers the energy barrier for pore opening and provides driving force. Thereby, tether proteins assume a critical mechanical role in the terminal stage of membrane fusion, which is likely to be conserved at multiple steps of vesicular traffic. SNAREs and tether proteins should hence be 
considered as a single, non-dissociable device to drive fusion. The core fusion machinery may then be larger and more complex than hitherto thought.

SNAREs dock membranes by stepwise assembly into 4-helix bundles. They exert mechanical force through their transmembrane domains (TMDs) ${ }^{1,2}$. This induces fusion of the outer leaflets (hemifusion), followed by inner leaflet fusion and pore formation. Pore formation can be preceded by full zippering of the 4-helix bundle ${ }^{6}$. Fusion is often studied with synaptic SNAREs, which use unique cofactors to fuse highly curved neurotransmitter vesicles with exquisite speed and temporal control ${ }^{7}$. The membranes in most other fusion reactions fuse more slowly, are much less curved, and their SNARE density is lower. Fusion driven solely by SNAREs becomes much less effective with increasing vesicle diameter and decreasing SNARE density ${ }^{8,9}$. Then, multi-subunit tether complexes become important. These facilitate membrane contact, associate with the SNARE-binding SM proteins ${ }^{10-12}$ and promote transSNARE pairing. We investigated whether tether complexes enhance the fusogenic potential of SNARE complexes, by increasing the force that SNAREs transmit to the bilayers, or by lowering the energy barrier for fusion pore formation.

HOPS is the tether complex for vacuole and lysosome fusion. Vacuoles from cells deleted for the Rab7-GTPase Ypt7 lack HOPS (Extended Data Fig. 1a) ${ }^{13}$. This prevents fusion and pairing between the vacuolar SNAREs Vam3, Vti1, Vam7 and Nyv1 $1^{14}$. Incubating these membranes with soluble, recombinant vacuolar $Q_{c}$-SNARE Vam7 (rVam7) allows to stimulate trans-SNARE pairing. Then, the reaction is independent of endogenous Vam7, which must otherwise be liberated by Sec18/NSF-dependent disruption of cis-SNARE complexes $^{15}$. Fusion can hence proceed without ATP, avoiding interference by the ATP-driven chaperone NSF, which disassembles SNARE complexes unless HOPS protects them ${ }^{14}$. To assay trans-SNARE pairing, we separately prepared vacuoles from ypt7 $\Delta$ or wildtype strains expressing Nyv1-HA or Vam3-myc. We mixed them in fusion reactions with rVam7 and measured 
trans-SNARE pairing through co-immunoadsorption of Vam3-myc with Nyv1HA. Adding rVam7 to ypt7 $\Delta$ vacuoles induced similar trans-SNARE pairing as in wildtype vacuoles (Fig. 1a,b). We measured content mixing by transfer of a $45 \mathrm{kDa}$ enzyme between the fusion partners (Extended Data Fig. 2), and lipid mixing by fluorescence dequenching through dilution of rhodaminephosphatidylethanolamine ${ }^{3,16}$. The trans-SNARE complexes on ypt7A vacuoles failed to induce content mixing (Fig. 1c), whereas lipid mixing was similar to wildtype (Fig. 1d,e). Thus, HOPS-free ypt7 $\Delta$ vacuoles reached a hemifused state but failed to form a fusion pore, or to open it wide enough to let the reporter pass. Lipid mixing was sensitive to antibodies targeting the $\mathrm{Q}_{\mathrm{a}}$ SNARE Vam3 or the R-SNARE Nyv1, confirming that the reaction was SNARE-dependent. The ypt7 $\Delta$ trans-SNARE complexes are probably fully zippered, because this is prerequisite for lipid mixing ${ }^{17}$.

To test the effect of HOPS on pore opening, we accumulated ypt7 $\Delta$ vacuoles for $60 \mathrm{~min}$ in the hemifused state, added purified HOPS or subcomplexes thereof (Extended Data Fig. 1b), and assayed content mixing after 15 min of further incubation. HOPS is a hexameric complex with a globular SNAREbinding domain, which contains the SNARE-binding SM-protein Vps33 and its interactor Vps16 (Fig. 1f) ${ }^{18}$. Vps33 (79 kDa) did not stimulate fusion (Fig. 1g), whereas the bigger Vps33-Vps16 subcomplex (159 kDa) rescued fusion to $80 \%$ of an untreated wildtype control, which had been incubated under standard fusion conditions with ATP. A Vps11-Vps39 subcomplex (240 kDa), representing the opposite end of HOPS without a SNARE interaction site, had no effect (Fig. 1g). As the biggest structure, HOPS (663 kDa) rescued ypt7 $\Delta$ fusion to wildtype level. The endosomal CORVET complex, which is of similar size as HOPS and shares Vps33 with it ${ }^{11}$, stimulated fusion as well as HOPS (Extended Data Fig. 3). The specificity of these complexes is hence restricted to their Rab-GTPase-dependent function in membrane tethering. HOPS had not enhanced trans-SNARE pairing beyond the level attained by the preincubation with rVam7 (Fig. 1a,b). It did not induce fusion in the absence of Vam7, nor upon pre-incubating the vacuoles with antibodies to Vam3 (Fig. 1g, Extended Data Fig. 4). Thus, rescue was SNARE-dependent. These results 
suggest an additional role of HOPS in fusion pore opening, which is independent of its known role in facilitating SNARE pairing ${ }^{12,19}$.

To test whether pore opening might be driven by increased SNARE complex volume, we accumulated hemifused ypt7 $\Delta$ vacuoles and added CBP-Vps33, which does not stimulate pore opening. When we tripled the effective molecular mass of Vps33 by adding a monoclonal antibody (150 kDa) to its CBP-tag, content mixing increased 5 -fold and reached $>50 \%$ of the wildtype signal (Fig. 2a). Antibodies did not stimulate fusion when Vps33 had been omitted, nor when Vps33 was used with a non-cognate HA-antibody. CBPVps33 alone decreased content mixing of wildtype vacuoles by $60 \%$ and this inhibition could be partially overcome by adding CBP-antibodies. This can be understood if CBP-Vps33 outcompetes endogenous HOPS for SNARE binding but by itself does not add sufficient mass to stimulate fusion.

Next, we replaced HOPS by artificial SNARE-binding proteins, using solely polyclonal antibodies to SNAREs (Fig. 2b). When added from the beginning of a reaction, SNARE antibodies interfere with trans-SNARE pairing and block fusion. To circumvent this block, we first accumulated ypt7 $\Delta$ vacuoles in hemifusion, with trans-SNARE complexes already formed. Now, anti-Nyv1 or anti-Vam3 became strong stimulators. They rescued content mixing to $50 \%$ when added individually and to $100 \%$ when added simultaneously. Wildtype vacuoles, which contain sufficient amounts of endogenous HOPS, were hardly stimulated by the antibodies. Thus, artificial SNARE ligands substitute for HOPS in fusion pore opening in vitro.

Since bivalent antibodies to SNAREs might promote fusion by clustering SNARE complexes around a fusion site, we generated monovalent $F_{a b}$ fragments from them (Extended Data Fig. 1c). These inhibited fusion and trans-SNARE pairing when added before docking (Fig. 2c,d), but they potently stimulated content mixing of hemifused vacuoles without affecting transSNARE pairing. Individual use of $F_{a b} S$ to either Vam3 or Nyv1 stimulated fusion weakly, probably becaus e $F_{a b S}$ are smaller (56 kDa) than lgGs (150 
$\mathrm{kDa}$ ) and their dimensions permit to place no more than two $\mathrm{F}_{\mathrm{abS}}$ along a SNARE domain. $F_{a b}$ to a single SNARE may therefore not add sufficient volume to SNARE complexes to drive pore opening. Crowding agents ${ }^{20}$, such as Ficoll 400, had no influence, suggesting that bulky SNARE complex ligands do not stimulate fusion by molecular crowding (Extended Data Figs. 5, 3). Thus, HOPS may promote fusion pore formation in vitro by increasing the volume of SNARE complexes.

We engineered an in vivo system for recruiting large ligands to SNAREs, using FKBP12 and FRB, two domains undergoing rapamycin-induced dimerization ${ }^{21}$. FKBP12 (12 kDa) was attached to the C-terminus of Vam7, which is close to the TMDs of the SNARE complex. FRB-GFP (38 kDa) was attached to Pfk1, a subunit of the octameric, soluble phosphofructokinase (850 kDa), which is unrelated to fusion (Fig. 3a, Extended Data Fig. 1d,e). Rapamycin-insensitive tor $1-1$ cells served as strain background to avoid side effects of rapamycin treatment through TOR signaling ${ }^{22}$. tor1-1 cells have multiple vacuoles under normal growth conditions. Rapamycin recruited Pfk1FRB-GFP to vacuoles within less than $10 \mathrm{~min}$ (Fig. 3b,c). These vacuoles fused, significantly reducing their number per cell. Recruitment, but not fusion, was observed when FKBP12 was separated from Vam7 by a 35 amino acid linker (Fig. 3c). Vam7 lacking FKBP12 induced neither recruitment nor fusion (Fig. 3b). Also an FRB-GFP chimera lacking phosphofructokinase did not provoke fusion (Extended Data Fig. 6).

HOPS recruitment controls re-fusion of vacuoles following hypertonic shock ${ }^{23}$. Hypertonic shock fragments yeast vacuoles in $<5 \mathrm{~min}$, Vps41 becomes phosphorylated and dissociates into the cytosol, likely together with HOPS ${ }^{24}$. Vacuole recovery requires Vps41 dephosphorylation, reassociation of HOPS with vacuoles and fusion. Whereas re-fusion requires $>60$ min in wildtype cells, rapamycin-induced recruitment of Pfk1-FRB-GFP to SNAREs provoked premature fusion of these vacuolar fragments, circumventing the physiological inactivation of HOPS (Extended Data Fig. 7). Fusion was not observed in cells lacking FKBP12 on Vam7, nor when rapamycin was omitted. 
We explored the influence of bulky SNARE ligands by coarse-grained molecular dynamics (Fig. 4a). HOPS features a SNARE-binding domain of approximately 12-14 nm diameter, which probably encapsulates the SNARE complex $^{11,18}$ (Fig. 1f). Binding a sphere of the size of this head region to SNAREs markedly affects the geometry of the hemifusion stalk (Fig. 4a). The stalk restrains the apposed membranes from separating, enforcing strong local curvature. We rationalized the acceleration of fusion from the apparent work (free energy) required to thin the hemifusion stalk (Fig. 4a,b and SI). Progression from hemifusion to pore opening decreases the mutual distance of the SNARE C-termini until they associate (Fig. 4a). The presence of a HOPS sphere $(14 \mathrm{~nm}$ ) halves the energetic cost of fusion pore opening (from $67 \mathrm{k}_{\mathrm{B}} \mathrm{T}$ to $34 \mathrm{k}_{\mathrm{B}} \mathrm{T}$ ) in a tension-less membrane system with 3 SNARE complexes (Fig. 4b, Extended Data Fig. 8). Pore nucleation now requires farless thinning of the stalk. We attribute the enhanced (non-leaky) fusion pore formation to (I) a partial, relative relaxation of the HOPS-induced curvature stress ( $\sim 30 \mathrm{kB}$ T; Fig. 4c, Extended Data Fig. 9), and (II) a geometrical advantage because of the pre-existing curvature (Extended Data Fig. 8). Smaller contributions can be made by an initial gain in SNARE pulling force (Extended Data Fig. 10). HOPS thus stimulates pore opening by steric effects on the site of hemifusion. These aspects are elaborated in supplementary discussion.

We can understand numerous unexplained findings from this perspective: (1) SNARE-associated tether protein complexes (Munc13 and its associated SM protein Munc18) are essential for fusion of synaptic vesicles. While this can reflect their role in SNARE complex assembly, Munc13/18 might also drive fusion itself ${ }^{25}$. Accordingly, mutations that reduce Munc18 affinity for SNAREs change fusion pore dynamics ${ }^{26}$. (2) Also mutating the yeast Munc18 homolog Sec1, which associates with the tether complex exocyst ${ }^{27}$, reduces exocytosis without reducing SNARE complex abundance ${ }^{28,29}$. (3) On vacuoles, mutations compromising Vps33 binding to vacuolar SNAREs impair content mixing more than lipid mixing and trans-SNARE pairing ${ }^{16}$ (4) HOPS also enhances 
liposome fusion more than trans-SNARE pairing 30 ; (5) binding to Sec17/ $\alpha$ SNAP and Sec18/NSF renders trans-SNARE complexes more fusogenic ${ }^{17,31}$.

Our simulations and experimental observations suggest that bulky SNAREligands can drive fusion irrespective of specific molecular properties, except for their size. Since SM proteins and tethering complexes are indispensable for fusion in multiple trafficking pathways ${ }^{11}$, we propose that the driving force that they can contribute to fusion is a critical and conserved feature of their function. In physiological membranes, SNAREs and the tether/SM protein system thus act as an integrated molecular machine, in which tether/SM proteins first facilitate SNARE pairing ${ }^{11}$ and then drive the very last step of fusion.

\section{Acknowledgements}

We thank Véronique Comte and Jieqiong Gao for the purification of proteins and antibodies. This work was supported by grants from the DFG (SFB 944, to $\mathrm{CU}$ ), and SNF and ERC to AM.

\section{Author Contributions:}

HJR conceived and interpreted simulation experiments and values derived from them. MDA conceived, performed and interpreted all other experiments. AM conceived the study and interpreted the results. AL and CU provided purified CORVET, HOPS and HOPS subcomplexes. All authors jointly wrote the paper.

\section{Author Information}

Reprints and permissions information is available at www.nature.com/reprint. The authors have no competing financial interests. Correspondence and requests for materials should be addressed to andreas.mayer@unil.ch 


\section{References:}

1. Gao, Y. et al. Single reconstituted neuronal SNARE complexes zipper in three distinct stages. Science 337, 1340-1343 (2012).

2. Zhang, X. et al. Stability, folding dynamics, and long-range conformational transition of the synaptic t-SNARE complex. Proc. Natl. Acad. Sci. U.S.A. 113, E8031-E8040 (2016).

3. Reese, C., Heise, F. \& Mayer, A. Trans-SNARE pairing can precede a hemifusion intermediate in intracellular membrane fusion. Nature 436, 410-414 (2005).

4. Chernomordik, L. V. \& Kozlov, M. M. Protein-lipid interplay in fusion and fission of biological membranes. Annu Rev Biochem 72, 175-207 (2003).

5. Cohen, F. S. \& Melikyan, G. B. The energetics of membrane fusion from binding, through hemifusion, pore formation, and pore enlargement. $J$ Membr Biol 199, 1-14 (2004).

6. Shin, J., Lou, X., Kweon, D.-H. \& Shin, Y.-K. Multiple conformations of a single SNAREpin between two nanodisc membranes reveal diverse pre-fusion states. Biochemical Journal 459, 95-102 (2014).

7. Rizo, J. \& Südhof, T. C. The Membrane Fusion Enigma: SNAREs, Sec1/Munc18 Proteins, and Their Accomplices-Guilty as Charged? Annu Rev Cell Dev Biol 28, 279-308 (2012).

8. Hernandez, J. M., Kreutzberger, A. J. B., Kiessling, V., Tamm, L. K. \& Jahn, R. Variable cooperativity in SNARE-mediated membrane fusion. PNAS 111, 12037-12042 (2014).

9. Baker, R. W. et al. A direct role for the Sec1/Munc18-family protein Vps33 as a template for SNARE assembly. Science 349, 1111-1114 (2015).

10. Orr, A., Wickner, W., Rusin, S. F., Kettenbach, A. N. \& Zick, M. Yeast vacuolar HOPS, regulated by its kinase, exploits affinities for acidic lipids and Rab:GTP for membrane binding and to catalyze tethering and fusion. Mol. Biol. Cell 26, 305-315 (2015).

11. Kuhlee, A., Raunser, S. \& Ungermann, C. Functional homologies in vesicle tethering. FEBS Lett 589, 2487-2497 (2015). 
12. Zick, M. \& Wickner, W. The tethering complex HOPS catalyzes assembly of the soluble SNARE Vam7 into fusogenic trans-SNARE complexes. Mol. Biol. Cell 24, 3746-3753 (2013).

13. Price, A., Seals, D., Wickner, W. \& Ungermann, C. The docking stage of yeast vacuole fusion requires the transfer of proteins from a cis-SNARE complex to a Rab/Ypt protein. J Cell Biol 148, 1231-1238 (2000).

14. Xu, H., Jun, Y., Thompson, J., Yates, J. \& Wickner, W. HOPS prevents the disassembly of trans-SNARE complexes by Sec17p/Sec18p during membrane fusion. EMBO J 29, 1948-1960 (2010).

15. Thorngren, N., Collins, K. M., Fratti, R. A., Wickner, W. \& Merz, A. J. A soluble SNARE drives rapid docking, bypassing ATP and Sec17/18p for vacuole fusion. EMBO J 23, 2765-2776 (2004).

16. Pieren, M., Schmidt, A. \& Mayer, A. The SM protein Vps33 and the tSNARE H(abc) domain promote fusion pore opening. Nat Struct Mol Biol 17, 710-717 (2010).

17. Schwartz, M. L. \& Merz, A. J. Capture and release of partially zipped trans-SNARE complexes on intact organelles. J Cell Biol 185, 535-549 (2009).

18. Bröcker, C. et al. Molecular architecture of the multisubunit homotypic fusion and vacuole protein sorting (HOPS) tethering complex. PNAS 109, 1991-1996 (2012).

19. Orr, A., Song, H., Rusin, S. F., Kettenbach, A. N. \& Wickner, W. HOPS catalyzes the interdependent assembly of each vacuolar SNARE into a SNARE complex. Mol. Biol. Cell 28, 975-983 (2017).

20. $\mathrm{Yu}, \mathrm{H}$. et al. Reconstituting Intracellular Vesicle Fusion Reactions: The Essential Role of Macromolecular Crowding. J Am Chem Soc 137, 12873-12883 (2015).

21. Haruki, H., Nishikawa, J. \& Laemmli, U. K. The anchor-away technique: rapid, conditional establishment of yeast mutant phenotypes. Mol Cell 31, 925-932 (2008).

22. Michaillat, L., Baars, T. L. \& Mayer, A. Cell-free reconstitution of vacuole membrane fragmentation reveals regulation of vacuole size and number by TORC1. Mol. Biol. Cell 23, 881-895 (2012).

23. Zieger, M. \& Mayer, A. Yeast vacuoles fragment in an asymmetrical 
two-phase process with distinct protein requirements. Mol. Biol. Cell 23, 3438-3449 (2012).

24. LaGrassa, T. J. \& Ungermann, C. The vacuolar kinase Yck3 maintains organelle fragmentation by regulating the HOPS tethering complex. $J$ Cell Biol 168, 401-414 (2005).

25. Carr, C. M. \& Rizo, J. At the junction of SNARE and SM protein function. Curr Opin Cell Biol 22, 488-495 (2010).

26. Fisher, R. J., Pevsner, J. \& Burgoyne, R. D. Control of fusion pore dynamics during exocytosis by Munc18. Science 291, 875-878 (2001).

27. Morgera, F. et al. Regulation of exocytosis by the exocyst subunit Sec6 and the SM protein Sec1. Mol. Biol. Cell 23, 337-346 (2012).

28. Hashizume, K., Cheng, Y.-S., Hutton, J. L., Chiu, C.-H. \& Carr, C. M. Yeast Sec1p functions before and after vesicle docking. Mol. Biol. Cell 20, 4673-4685 (2009).

29. Grote, E., Carr, C. M. \& Novick, P. J. Ordering the final events in yeast exocytosis. J Cell Biol 151, 439-452 (2000).

30. Zick, M. \& Wickner, W. T. A distinct tethering step is vital for vacuole membrane fusion. elife 3, e03251 (2014).

31. Zick, M., Orr, A., Schwartz, M. L., Merz, A. J. \& Wickner, W. T. Sec17 can trigger fusion of trans-SNARE paired membranes without Sec18. PNAS 112, E2290-E2297 (2015).

32. Risselada, H. J., Bubnis, G. \& Grubmüller, H. Expansion of the fusion stalk and its implication for biological membrane fusion. 111, 1104311048 (2014). 


\section{Figure Legends}

\section{Figure 1: Effect of soluble Vam7 on lipid and content mixing.}

a, b, Trans-SNARE pairing. Vacuoles were isolated from wildtype (BJ3505) or isogenic ypt7 $\Delta$ cells carrying Vam3-myc or Nyv1-HA. The two vacuole populations were mixed and incubated in fusion reactions with ATP, Vam7 and HOPS as indicated. Proteins were solubilized, pulled down with anti-HA and analyzed by SDS-PAGE and Western blotting (a). Trans-SNARE pairing is assessed by Vam3myc co-adsorbed to Nyv1-HA. Bands were quantified (b). c-e, Hemifusion in ypt7A vacuoles: Vacuoles from wildtype and ypt7 $\Delta$ cells were incubated in ATP-free fusion reactions with $600 \mathrm{nM} \mathrm{rVam} 7$ and $10 \mathrm{mg} / \mathrm{ml} \mathrm{BSA}$. Antibodies (200 nM) had been added where indicated. c, Content mixing was determined after 60 min, using the activation of pro-alkaline phosphatase by a maturase from the fusion partners. d,e, Lipid mixing was followed by dequenching of the fluorescence of Rhphosphatidylethanolamine, which had been integrated at self-quenching concentrations into one of the fusion partners. $\mathbf{f}$, Structure of HOPS (from ${ }^{18}$ ). $\mathbf{g}$, Effect of HOPS subcomplexes on content mixing. Vacuoles were incubated in fusion reactions as in $\mathrm{C}$ for $60 \mathrm{~min}$, with anti-Vam3 where indicated. Then, the samples received $400 \mathrm{nM}$ purified (CBP)-tagged HOPS sub-complexes or Vps33 and were incubated for further 15 minutes before content mixing was assayed. For a-e and $\mathbf{g}$, means \pm s.d. are shown from $n=3$ biologically independent experiments.

Figure 2: Fusion pore opening driven by ligands increasing SNARE complex size in vitro.

a, Vps33. Fusion reactions with wildtype and ypt7 $\Delta$ vacuoles were started as in Fig. 1g. After the first $60 \mathrm{~min}$ incubation period, samples received recombinant CBPVps33 (400nM), antibodies to CBP or HA (200 nM), or buffer only. After further 15 min, content mixing was assayed. b, Antibodies. Two-stage fusion reactions were performed as in a, but only with rVam7. Antibodies (200 nM) against Vam3, Nyv1 or Sec18/NSF were added either during the first 60 min incubation $\left(1^{\circ}\right)$ or during the second incubation $\left(\mathrm{II}^{\circ}\right)$ of $15 \mathrm{~min}$. c, $\mathrm{F}_{\mathrm{ab}}$ fragments. Experiment as in b, but with $F_{a b}$ fragments instead of antibodies. $d$, Trans-SNARE pairing was assayed using tagged strains as in Fig. 1a. Reactions with staged addition of rVam7 and $F_{a b S}$ 
were run as in c. For all subfigures, means \pm s.d. are shown from $n=3$ biologically independent experiments.

Figure 3: Effect of SNARE complex enlargement on vacuole fusion in vivo. a, Schematic view of rapamycin-induced FKBP12/FRB-tagged Pfk1-recruitment to the SNARE complex without and with a long linker between Vam7 and FKBP12. b, c, In vivo vacuole morphology. Logarithmically growing cells, carrying Pfk1-FRBGFP and (b) Vam7 or Vam7-2xFKBP12, or (c) Vam7-LL-2xFKBP12 with a 35 amino acid linker, were stained with the vacuole tracer FM4-64. Cells were incubated with $10 \mu \mathrm{M}$ rapamycin for $10 \mathrm{~min}$ where indicated and analyzed by spinning disc microscopy. The cells were grouped into three categories according to the number of vacuoles visible per cells. 100 cells were analyzed per sample. Scale bar: $5 \mu \mathrm{m}$. Means and s.d. are shown from $n=3$ biologically independent experiments.

Figure 4: Molecular dynamics simulations on the influence of steric constraints at the (hemi-)fusion site.

a, Simulation setup: Two hemifused membranes in the presence of the SNARE complex that is either free or bound to a sphere equivalent to the size of the SNARE-binding domain of HOPS (14 nm). The size of 'HOPS' and positioning of the SNARE complex are inspired by EM and crystal structures ${ }^{9,11,18}$. To enhance clarity, only one SNARE complex is illustrated. $\mathbf{b}$, The free-energy of fusion pore formation is derived by measuring the work (free energy) required to thin the stalk until fusion pore nucleation occurs ${ }^{32}$ (see Extended Data Fig. 8). Arrows and dotted lines indicate the point of pore nucleation. Error bars are calculated via Bayesian resampling of 50 overlapping WHAM histograms. Each parental WHAM histogram is comprised of $>30000$ data points (autocorrelation up to $\sim 1500$ data points). c, Work distribution reflecting the equilibrium work that HOPS must perform to bend the membrane in the presence of a stalk (average work: 115+/- 2 $k_{B} T$ ) or of a fusion pore (average work: $88+/-3 k_{B} T$ ), respectively. A reduction of $\sim 30 \mathrm{k}_{\mathrm{B}} \mathrm{T}$ indicates that fusion pore formation is associated with release of local membrane stress. 


\section{Methods}

\section{Strains and culture conditions}

All strains were grown in either in YPD (yeast extract, peptone, dextrose) containing $2 \%$ glucose in the presence or absence of G418, or in SC (synthetic dextrose) dropout media containing 2\% glucose to select for auxotrophies. Strains used in this study can be found in Table S1 in supplementary information. Primers used can be found in Table S2. Vam7-LL2xFKBP12 contains a linker (LL) of 35 amino acids with the sequence SGGGGSGGGG SGGGGSGGGG SGGGGSGGGG GAAGG.

Genetic manipulations: Yeast transformations were carried out using the lithium acetate method. Gene deletions and tagging were performed as previously established ${ }^{33,34}$. Genome-tagging of Vam7 with 2xFKBP12 and LL2xFKBP12 was performed starting from the plasmid pTK209, from which GFP was removed by double digestion with $\mathrm{Pacl}$ and Ascl restriction enzymes and replaced by a $2 x F K B P 12$ coding sequence carrying the same restriction sites. The 2xFKBP12 sequence was obtained by gene synthesis (BIOCAT) and cloned into a pUC57 vector. pRS415-TEFpr-FRB-GFP was obtained starting from a pRS416-S3-FRB-GFP vector (provided by C. Ungermann's group). The FRB-GFP coding frame was amplified by PCR using the primers reported above and cloned into the pRS415-TEFpr vector using HindIII and Sacl restriction sites.

\section{Vacuole isolation}

BJ3505 and DKY6281 strains carrying tagged SNAREs were grown in YPD at $\left(30^{\circ} \mathrm{C}, 225 \mathrm{rpm}\right)$ to $\mathrm{OD}_{600}=1$ and harvested $\left(3 \mathrm{~min}, 5^{\prime} 000 \mathrm{xg}\right)$. Harvested cells were resuspended in reduction buffer (30 mM Tris/Cl pH 8.9, $10 \mathrm{mM}$ DTT) and incubated for $5 \mathrm{~min}$ at $30^{\circ} \mathrm{C}$. After harvesting as described above, cells were resuspended in $15 \mathrm{ml}$ digestion buffer (600 mM sorbitol, $50 \mathrm{mM} \mathrm{K-}$ phosphate $\mathrm{pH} 7.5$ in YP medium with $0.2 \%$ glucose and $0.1 \mathrm{mg} / \mathrm{ml}$ lyticase preparation). After $25 \mathrm{~min}$ at $30^{\circ} \mathrm{C}$, cells were centrifuged (2 min, 5'200 rpm, JLA25.5 rotor). The spheroblasts were resuspended in $2 \mathrm{ml} \mathrm{15 \%} \mathrm{Ficoll-400} \mathrm{in}$ PS buffer (10 mM PIPES/KOH pH 6.8, 200 mM sorbitol) and $150 \mu \mathrm{l}$ (for 
DKY6281-derived strains) or $250 \mu \mathrm{l}$ (for BJ3505 derivatives) DEAE dextran $\left(0.4 \mathrm{mg} / \mathrm{ml}\right.$ in PS). After $2 \mathrm{~min}$ of incubation at $30^{\circ} \mathrm{C}$, the cells were transferred to SW41 tubes and overlaid with steps of $8 \%, 4 \%$ and $0 \%$ Ficoll-400 in PS.

Cells were centrifuged for $90 \mathrm{~min}$ at $4^{\circ} \mathrm{C}$ and $301000 \mathrm{rpm}$ in a SW41 rotor. Lyticase had been recombinantly expressed in E.coli RSB805 (provided by Dr. Randy Schekman, Berkeley) and prepared from a periplasmic supernatant ${ }^{3}$.

\section{Vacuole fusion and content mixing assay}

DKY6281 and BJ3505 vacuoles were adjusted to a protein concentration of $0.5 \mathrm{mg} / \mathrm{ml}$ and incubated in a volume of $30 \mu \mathrm{PS}$ buffer (10 mM PIPES/KOH $\mathrm{pH}$ 6.8, $200 \mathrm{mM}$ sorbitol) with $125 \mathrm{mM} \mathrm{KCl}, 0.5 \mathrm{mM} \mathrm{MnCl}_{2}$. Note that the harvested vacuole suspension contains around $20 \mathrm{mg} / \mathrm{ml}$ Ficoll 400, creating an environment of moderate molecular crowding ${ }^{35}$. Vacuoles were preincubated with inhibitors on ice ( $5 \mathrm{~min}$ ) before starting the fusion by addition of the ATP-regenerating system $(0.25 \mathrm{mg} / \mathrm{ml}$ creatine kinase, $20 \mathrm{mM}$ creatine phosphate, $500 \mu \mathrm{M}$ ATP, $500 \mu \mathrm{M} \mathrm{MgCl}_{2}$ ) or of $600 \mathrm{nM} \mathrm{rVam7}$ and 10 $\mathrm{mg} / \mathrm{ml} \mathrm{BSA}$. Samples were incubated for $60 \mathrm{~min}$ at $27^{\circ} \mathrm{C}$. In two-stage reactions, a second incubation of 15 at $27^{\circ} \mathrm{C}$ was added, with $200 \mathrm{nM}$ of antibodies or $400 \mathrm{nM}$ of purified HOPS subunits. In order to assay fusion, $1 \mathrm{ml}$ of PS buffer was added, vacuoles were centrifuged ( $2 \mathrm{~min}, 20^{\prime} 000 \mathrm{xg}, 4^{\circ} \mathrm{C}$ ) and resuspended in $500 \mu$ leveloping buffer $\left(10 \mathrm{mM} \mathrm{MgCl}_{2}, 0.2 \% \mathrm{TX}-100\right.$, $250 \mathrm{mM}$ Tris- $\mathrm{HCl} \mathrm{pH} \mathrm{8.9,} 1 \mathrm{mM}$ p-nitrophenylphosphate). After $5 \mathrm{~min}$ at $27^{\circ} \mathrm{C}$, the reactions were stopped with $500 \mu \mathrm{l} 1 \mathrm{M}$ glycine $\mathrm{pH} 11.5$ and the OD was measured at $405 \mathrm{~nm}$. Background activity of pro-Pho8 was assessed through a fusion sample kept on ice throughout the incubation period. The value of this sample was subtracted from the others.

\section{Lipid mixing assay}

Lipid mixing was assayed as described ${ }^{3}$. In brief, $30 \mu \mathrm{g}$ of unlabeled BJ3505 vacuoles and $6 \mu \mathrm{g}$ of rhodamine-labeled phosphoethanolamine DKY6281 vacuoles were mixed in $190 \mu \mathrm{l}$ of $0.3 \mathrm{mM} \mathrm{MnCl}_{2}, 75 \mathrm{mM} \mathrm{KCl}$ in PS buffer. Inhibitors were pre-warmed to $27^{\circ} \mathrm{C}$ before being adding to the tubes. Fusion reactions were started by adding $9.5 \mu \mathrm{l}$ of $20 x$ ATP-regeneration system, yielding $0.125 \mathrm{mg} / \mathrm{ml}$ creatine kinase, $20 \mathrm{mM}$ creatine phosphate, $0.5 \mathrm{mM}$ 
ATP, $0.5 \mathrm{mM} \mathrm{MgCl}_{2} .100 \mu \mathrm{l}$ were used to assay lipid mixing in a fluorescent plate reader at $27^{\circ} \mathrm{C}$ for $32 \mathrm{~min} .80 \mu \mathrm{l}$ were incubated separately for $60 \mathrm{~min}$ and then assayed for content mixing by alkaline phosphatase developing buffer as described above.

\section{Immunoprecipitations}

Vacuoles from a $1 \mathrm{ml}$ fusion reaction were pelleted $\left(5 \mathrm{~min}, 6^{\prime} 000 \mathrm{xg}, 4^{\circ} \mathrm{C}\right)$, solubilized for $10 \mathrm{~min}$ in lysis buffer (0.5\% Triton X-100, $0.5 \mathrm{mM} \mathrm{MnCl}, 100$ $\mathrm{mM} \mathrm{CaCl} 2,1 \mathrm{mM} \mathrm{PMSF}$ ) and centrifuged for $10 \mathrm{~min}$ at $12^{\prime} 000 \mathrm{xg}$ and $4^{\circ} \mathrm{C}$. The supernatant was supplemented with $30 \mu \mathrm{g}$ of antibody and $25 \mu \mathrm{l}$ of protein-G sepharose and shaken for $60 \mathrm{~min}$ at $4^{\circ} \mathrm{C}$. The beads were washed three times with lysis buffer and suspended in SDS sample buffer.

\section{Gel electrophoresis and Western blot}

Protein samples were dissolved in reducing sample buffer and heated to $95{ }^{\circ} \mathrm{C}$ for 5 minutes. The samples were run on either $10 \%$ or $12.5 \%$ polyacrylamide gels. The stacking gels were prepared as follows: $6 \%$ acrylamide, $0.16 \%$ bis-acrylamide, $0.1 \mathrm{M}$ Tris $\mathrm{pH} 6.8,0.1 \%$ SDS, $0.1 \%$ TEMED, $0.05 \%$ ammonium persulfate. Running gels were: $10 \%$ or $12.5 \%$ acrylamide, $0.27 \%$ or $0.34 \%$ bis-acrylamide, $0.38 \mathrm{M}$ Tris $\mathrm{pH} 8.8,0.1 \%$ SDS, $0.06 \%$ TEMED, $0.06 \%$ APS. The gels $(10 \mathrm{~cm} / 8 \mathrm{~cm} / 1.5 \mathrm{~mm})$ were run at constant current (20-30 mA). Proteins were blotted onto nitrocellulose membrane by the semidry method for $80 \mathrm{~min}$ at $400 \mathrm{~mA}$. After incubation with the primary antibody overnight, signals were detected by secondary antibodies coupled to infrared dyes and detected on a LICOR Odyssey infrared laser scanner. The files were exported as TIFF and processed in adobe illustrator CS3. Band intensity was quantified using densitometry software supplied with the Odyssey Infrared Imager.

\section{FM4-64 staining}

Cells were inoculated from a pre-culture in stationary phase and grown overnight to logarithmic phase $\left(\mathrm{OD}_{600}\right.$ between 0.2 and 0.8$)$. After dilution to an $\mathrm{OD}_{600}$ of 0.2 in $1 \mathrm{ml}$ culture, FM4-64 (N-(3-Triethylammoniumpropyl)-4-(6(4-(Diethylamino) Phenyl) Hexatrienyl) Pyridinium Dibromide) in DMSO was 
added to a final concentration of $10 \mu \mathrm{M}$. Cells were stained for $1 \mathrm{~h}$, followed by three washing steps in medium without stain (2 min, 3'000 xg) and a subsequent chase of 1 to $2 \mathrm{~h}$ in medium without stain, depending on the endocytotic capacity of the strain. The cells for microscopy were grown at $30^{\circ} \mathrm{C}$. The temperature was kept constant during staining and visualization. Care was taken to analyze cells immediately after their removal from the culture tube.

\section{Antibodies and affinity purification}

Sources of monoclonal antibodies were: anti-HA (16B12, MMS-101P, Covance); anti-myc (9E11, sc-47694, Santa Cruz Biotechnology). Polyclonal antibodies against Vam3, Nyv1, Vam7, Vps39, Ypt7 and GFP had been raised by injecting purified recombinant hydrophilic parts of these proteins into rabbits. Antibodies were purified from sera. Sera were first heated for 30 minutes at $56^{\circ} \mathrm{C}$ to inactivate the complement system, diluted $1: 1$ in PBS and filtered through $0.2 \mu$ membranes before being passed onto an activated $\mathrm{CH}$ sepharose 4B column (GE Healthcare Life Sciences 17-0430-01), which had been coupled with the recombinant protein of interest, according to the instructions of the manufacturer. The column was washed with 10 bed volumes of PBS at $4^{\circ} \mathrm{C}$. The antibodies were eluted with $0.2 \mathrm{M}$ glycine- $\mathrm{HCl} \mathrm{pH}$ $2.5,4^{\circ} \mathrm{C}$, using a peristaltic pump. Eluted fractions were collected on ice in 1,5 $\mathrm{ml}$ tubes containing $150 \mu \mathrm{l}$ of $1 \mathrm{M}$ Tris $\mathrm{pH} 8.8$ in order to neutralize the samples immediately. Protein concentration in the sample was determined by Bradford assay using BSA as a standard. Fractions of interest were pooled, transferred into PS buffer (10 mM PIPES-KOH pH 6.8, 200 mM sorbitol) containing $150 \mathrm{mM} \mathrm{KCl}$ by repeated dilution and re-concentration in Amicom®Ultra-15 30K ultrafiltration devices (Millipore). The antibodies were finally concentrated to $1-3 \mathrm{mg} / \mathrm{ml}$, aliquoted, flashed frozen in liquid nitrogen and kept at $-20^{\circ} \mathrm{C}$.

\section{Papain digestion and $F_{a b}$ fragment purification}

Antibody digestion with papain was described previously ${ }^{36}$. Briefly, $10 \mathrm{mg}$ affinity-purified IgG were solubilized in $1 \mathrm{ml}$ buffer $\mathrm{A}(150 \mathrm{mM} \mathrm{NaCl}, 1 \mathrm{mM}$ EDTA, $25 \mathrm{mM}$ mercaptoethanol, $10 \mathrm{mM} \mathrm{NaP}$ pH 7.3), followed by addition of 
$0.1 \mathrm{mg}$ papain and incubation for $3 \mathrm{~h}$ at $37^{\circ} \mathrm{C} .30 \mathrm{mM}$ iodoacetamide was added to inhibit papain $\left(15 \mathrm{~min}\right.$ at $\left.37^{\circ} \mathrm{C}\right)$. Afterwards, the sample was chilled to $4^{\circ} \mathrm{C}$ and loaded on a protein $\mathrm{A}$ agarose column (Pharmacia; $1.5 \mathrm{ml}$ volume), which was equilibrated before with buffer $\mathrm{B}\left(100 \mathrm{mM} \mathrm{KP}, \mathrm{pH}\right.$ 8.0). The $\mathrm{F}_{\mathrm{ab}}$ containing flow-through was dialyzed against $\mathrm{H}_{2} \mathrm{O}$ and concentrated by ultrafiltration through $30 \mathrm{kDa}$ cutoff membranes (Millipore).

\section{Purification of rVam7}

Plasmid pGEX-KT::Vam7 (kind gift from A. Merz, Seattle) was expressed in Rosetta 2 (DE3) (Novagen). Bacteria were grown in 2 I LB with $100 \mu \mathrm{g} / \mathrm{ml}$ ampicillin and $25 \mu \mathrm{g} / \mathrm{ml}$ chloramphenicol to $\mathrm{OD}_{600}=1$, induced with $1 \mathrm{mM}$ IPTG, $30^{\circ} \mathrm{C}$ for $4 \mathrm{~h}$. Cells were harvested and washed with PBSEEG (2 mM EDTA, 1 mM EGTA, 2 mM DTT, 1 mM PMSF in 1X PBS). The pellet was frozen in liquid nitrogen and stored at $-80^{\circ} \mathrm{C}$. For purification the sample was thawed, resuspended in $40 \mathrm{ml}$ PBSEEG, sonicated $(2 \times 1$ min on ice with maximal intensity), centrifuged (TI-60 rotor, $64^{\prime} 000 \times \mathrm{g}, 30 \mathrm{~min}, 4^{\circ} \mathrm{C}$ ) and the supernatant was incubated with 2-3 ml glutathion-sepharose 4B (GE Healthcare, 17-0756-01) under gentle rotation overnight, at $4^{\circ} \mathrm{C}$ and washed 3-4 $\mathrm{x}$ with PBSEEG. The resin was poured into a $10 \mathrm{ml}$ polypropylene column (Thermo scientific $\mathrm{N}^{\circ} 2994$ ), washed with 10 bed volumes of thrombin cleavage buffer (50 mM Tris- $\mathrm{HCl} \mathrm{pH} 8.0,100 \mathrm{mM} \mathrm{NaCl}, 2.5 \mathrm{mM} \mathrm{CaCl}_{2}, 0.1 \%$ $\beta$-mercaptoethanol) at room temperature. 200 Units of thrombin (Sigma T1063, $1000 \mathrm{U}$, dissolved in $0.5 \mathrm{ml}$ thrombin cleavage buffer and $0.5 \mathrm{ml}$ glycerol, aliquots had been kept at $-20^{\circ} \mathrm{C}$ ) were then added directly onto the column, the column was closed on both ends and incubated for 30 minutes at room temperature with end over end rotation. A second column with $1 \mathrm{ml} \mathrm{p}$ aminobenzamidine agarose (Sigma A-8332, $5 \mathrm{ml}$ ) was washed with $20 \mathrm{ml}$ thrombin cleavage buffer. The glutathione sepharose column was eluted with thrombin cleavage buffer directly onto this second column. Fractions of the flow-through were collected at the bottom of the second column. Protein levels were measured and fractions of interest were pooled. Eluted protein was transferred into PS buffer (10 mM PIPES-KOH pH 6.8, 200 mM sorbitol) containing $150 \mathrm{mM} \mathrm{KCl}$ by repeated dilution and ultrafiltration in Amicon Ultra- 
$1530 \mathrm{~K}$ (Millipore), finally concentrated to $3 \mathrm{mg} / \mathrm{ml}$, aliquoted, flash-frozen in liquid nitrogen and kept at $-20^{\circ} \mathrm{C}$.

\section{Purification of HOPS, HOPS subcomplexes and CORVET}

These complexes were purified via the TAP protocol, as previously described (Ostrowicz, Brocker et al., 2010). In brief, yeast cell lysates were prepared from $500 \mathrm{OD}_{600}$ equivalents of cells by thoroughly vortexing cells in lysis buffer [50 mM HEPES/KOH, pH 7.4, 300 mM NaCl, 0.15\% NP-40 (Igepal CA-630; Sigma-Aldrich), $2 \mathrm{mM} \mathrm{MgCl}$, $1 \mathrm{mM}$ DTT, $1 \mathrm{mM}$ phenylmethylsulphonyl fluoride (PMSF) and 1XFY protease inhibitor mix (Serva)] together with glass beads in a Disrupter Genie for $10 \mathrm{~min}$ at $2^{\circ} \mathrm{C}$, followed by centrifugation at $20^{\prime} 000 \mathrm{xg}$ at $4^{\circ} \mathrm{C}$. The supernatant was centrifuged for $60 \mathrm{~min}$ at $100^{\prime} 000 \mathrm{xg}$ and the cleared lysate loaded onto $25 \mu$ of prewashed lgG beads. After $1 \mathrm{~h}$ of incubation at $4^{\circ} \mathrm{C}$, the beads were washed 3 times with $1 \mathrm{ml}$ lysis buffer containing $0.5 \mathrm{mM}$ DTT, but lacking protease inhibitors. Bound proteins were eluted by TEV protease treatment for $1 \mathrm{~h}$ at $16^{\circ} \mathrm{C}$. TEV eluates were either analyzed by SDS-PAGE and Coomassie staining or loaded onto $25 \mu \mathrm{l}$ of prewashed calmodulin-sepharose beads, and incubated for $1 \mathrm{~h}$ at $4^{\circ} \mathrm{C}$. The beads were washed 3 times with $1 \mathrm{ml}$ lysis buffer, bound proteins were eluted by incubation with $20 \mathrm{mM}$ EGTA in lysis buffer for $20 \mathrm{~min}$ at $30^{\circ} \mathrm{C}$, analyzed by SDS-PAGE and Coomassie staining and kept in small aliquots at $-20^{\circ} \mathrm{C}$. Aliquots were thawed and the proteins transferred into lysis buffer without DTT and NP-40 by repeated dilution and ultrafiltration $\left(4^{\circ} \mathrm{C}\right)$ in Amicon Ultra$1530 \mathrm{~K}$ (Millipore). Re-concentrated proteins were used immediately for the experiment and not re-frozen.

\section{Rapamycin-induced protein re-localisation}

Cells were grown in YPD over night at $30^{\circ} \mathrm{C}$ to early logarithmic phase. Cells were diluted to $\mathrm{OD}_{600}=0.2$ for staining with $5 \mu \mathrm{M}$ FM4-64 and then incubated with rapamycin $(10 \mu \mathrm{M})$ before image acquisition.

\section{Statistics for biological experiments}

When data was averaged, the samples stem from independent experiments with independent preparations of vacuoles or cells, i.e. they represent 
biological replicates. The number of replicates is indicated in all figures as $n$, the variation of their values is characterized by the standard deviation (s. d.). Significance of differences has been evaluated through Student's t-test. Differences are only mentioned as such and interpreted if $p<0.005$.

\section{Molecular dynamics}

\section{Simulation model and settings}

The molecular dynamics simulations were performed with the GROMACS simulation package ${ }^{37}$, version 4.5.7. We used the MARTINI coarse-grained model 38,39 to simulate the lipids, amino acids and solvent. In all simulations, the system was coupled to a constant temperature bath using the 'V-rescale' algorithm with a relaxation time of $1.0 \mathrm{ps}$. All simulations were performed at a temperature of $293 \mathrm{~K}$. Periodic boundary conditions where applied to simulate bulk behavior. The time step used in the simulation was $20 \mathrm{fs}$. The dielectric constant in the simulations was $\varepsilon_{r}=15$. The neighbor-list was updated every 10 simulation steps. The pressure was weakly coupled ${ }^{40}$ to 1 bar with a relaxation time of $1.0 \mathrm{ps}$. Here, only the z-dimension was independently coupled to the pressure bath because the $\mathrm{x}$ and $\mathrm{y}$-dimension of the simulation box were conserved (see Membrane simulation setups).

\section{Modeling the vacuolar SNARE complex}

The vacuole SNARE complex was modeled using the MARTINI model for proteins ${ }^{38}$, which qualitatively captures the chemical nature of each individual amino acid and includes the secondary structure. For NYV1 the modeled sequence is "IGDATEDQIKDVIQIMNDNIDKFLERQERVSLLVDKTSQLNSSSNKFRRKAV NIKEIMWW[QKVKN]ITLLTFTIILFVSAAFMFFYLW", for VAM3:

"TIIHQERSQQIGRI

HTAVQEVNAIFHQLGSLVKEQGEQVTTIDENISHLHDNMQNANKQLTRA[DQ HQRDRNK]CGKVTLIIIIVVCMVVLLAVLS", for VTI1: "IDDDQRQQLLSNHAILQKSG 
DRLKDASRIANETEGIGSQIMMDLRSQRETLENARQTLFQADSYVDKSIKTL KTMTR[RLVANK]FISYAIIAVLILLILLVLFSKFK", and for VAM7 "MQMVRDQEQELV

ALHRIIQAQRGLALEMNEELQTQNELLTALEDDVDNTGRRLQIANKKARHF”. Here, the brackets [] depict the defined juxta-membrane (linker) regions. The resolved and previously simulated structure ${ }^{40}$ of the neuronal SNARE complex was used as a template structure for the vacuolar SNARE complex. To this aim, we applied an external field, using a self-modified version of Gromacs, to drive the structure of the vacuole SNARE complex toward the known structure of the neuronal SNARE complex based on the known alignment. All residues are defined alpha-helical except for the defined SNARE linkers. The linkers are either modeled as a random coil ' $\sim$ ' (in case of unstructured) or alpha helical ' $\mathrm{H}$ ' (in case of structured). We modeled all of the three SNARE linkers as being structured because such a scenario maximizes the force which a partly-assembled SNARE complex, by itself, can exert on the formed stalk.

\section{Membrane simulation setups}

In total three different 'HOPS' systems were simulated:

(1) A system with 3 SNARE complexes (10'158 POPC, 6'771 POPE, 663'000 water molecules, dimension $\left.48 \times 64 \times 37 \mathrm{~nm}^{3}, \mathrm{t}_{\mathrm{eq}}=1 \mu \mathrm{s}\right)$, used in Fig. $4 \mathrm{~b}$ and Extended Data Fig. 8);

(2) A system with 1 SNARE complex (10'983 POPC, 400'000 water molecules, dimension $\left.48 \times 40 \times 33 \mathrm{~nm}^{3}, \mathrm{t}_{\mathrm{eq}}=1 \mu \mathrm{s}\right)$, used in Fig. 4a, Extended Data Figs. 9b, 10);

(3) A system with 1 SNARE complex (13'621 POPC, 500'000 water molecules, dimension 67x40x40 nm ${ }^{3}, \mathrm{t}_{\mathrm{eq}}=2 \mu \mathrm{s}$ ), used in Fig. 4a, Extended Data Fig. 9b). For studying the fusion pore, the system was made slightly larger in order to avoid a too close distance between the fusion pore and the free membrane edges (see the description below).

It is important to emphasize that the two opposing membranes must be able to freely adopt their (local) separation distance in order to realistically mimic a scenario where two vacuoles fuse. To this aim, we cut the periodicity of the 
membrane along the x-dimension (thus preventing that solvent is 'trapped' in the space between the two opposing leaflets). The latter creates four free membrane edges (e.g., see Extended Data Fig. 8) which facilitate rapid flipflop between the leaflets and thereby ensure that the spontaneous curvature of the membrane vanishes when the membrane is bent (preventing finite size effects). Furthermore, the ability to freely adapt the area of the membrane ensures that the membrane minimizes its shape under tension-less conditions in the presence of HOPS and thus the work performed by HOPS is only determined by membrane bending energy. Finally, to prevent that the large line tension of the free membrane edges would strongly deform the simulation box (it prefers to minimize the $y$-dimension while maximizing the $x$-dimension) the $x$ - and $y$ dimension of the simulation box were kept constant. Hence, pressure coupling along these dimensions is redundant for a membrane cut in one dimension because the membrane area can independently adjust with respect to the (corresponding) area of the simulation box (the system is isotropic).

\section{Model and setup of the HOPS simulations}

In our simulations, 'soluble' HOPS is modeled by a soft harmonic repulsive potential $\left(\mathrm{K}_{\text {force }}=50 \mathrm{~kJ} \mathrm{~nm}^{-2} \mathrm{~mol}^{-1}\right)$. The 'attractive' HOPS is modeled by the potential function, $V(d)=K_{\text {force }} d^{2}\left(d^{2}-C\right)$, where $V(d)$ is the potential energy as a function of the penetration depth d, i.e. the distance beyond the surface of 'HOPS'. Here, Kforce and C (the width of the well) are set to $\mathrm{K}_{\text {force }}=20 \mathrm{~kJ} \mathrm{~nm}^{-}$ ${ }^{2} \mathrm{~mol}^{-1}$ and $\mathrm{C}=0.4 \mathrm{~nm}^{2}$. Because the additional presence of attractions reduces the apparent radius of HOPS we compensated for this by slightly increasing its radius $(7.4 \mathrm{~nm}$ versus $7.0 \mathrm{~nm})$. The 'HOPS' potential only acted on the carbon tails and glycerol parts of the lipids. To mimic a SNARE complex which is slightly embedded by HOPS ${ }^{11}$ we modeled a slight overlap between HOPS and the SNARE complex, i.e. the SNARE complex is located within HOPS about $2 \mathrm{~nm}$ away from its surface (see Extended Data Figs. 8/10). In reality, the depletion of solvent interactions upon binding will be compensated by competitive interactions with the binding pocket of HOPS. In our model, solvent was allowed to freely enter and pass 'HOPS' in order to conserve ongoing interactions within the coiled-coil complex of the SNAREs. 
The main advantage of modeling HOPS by an effective potential is that this allows direct quantification of the work which HOPS must perform to conserve the corresponding membrane shape of minimal free energy. The HOPS simulations were setup via the slow growth method, i.e., the radius of HOPS was gradually increased from 0 to the target radius over $80 \mathrm{~ns}$. During this procedure, the SNARE complex was restrained by restraining only a single bead within the SNARE complex (the backbone bead of residue GLY218 within VAM3) via a harmonic potential $\left(\mathrm{K}_{\text {force }}=1000 \mathrm{~kJ} \mathrm{~nm}^{-2} \mathrm{~mol}^{-1}\right)$. After equilibrium was reached - equilibrium was characterized by the pressure (bending work) and the resultant force on HOPS - we restrained 15 additional backbone atoms within VAM3 (GLY218 - ASP232) to simulate a torsional restraining effect of the binding pocket on the SNARE complex.

Finally, the stalk in all of the setups is generated by applying an external field. Here, we applied a harmonic potential $\left(50 \mathrm{~kJ} \mathrm{~nm}^{-2} \mathrm{~mol}^{-1}\right)$ to induce a cylindrically shaped 'void' of $1.0 \mathrm{~nm}$ radius in the solvent layer between the bilayers. The hydrophobic nature of the void attracts the lipid tails in the adjacent leaflets and results in the formation of a stalk. Notable, this whole process occurs on a timescale of a few nanoseconds only. The external potential is removed prior to subsequent equilibration and introduction of the HOPS sphere.

\section{Free energy of fusion and SNARE forces}

We performed two different types of so-called umbrella sampling protocols with different goals:

(A) How does HOPS binding affect the free energy landscape of fusion pore opening? To this end, we used simulation system 1 (see above) with probe to probe distance (stalk thickness) as a reaction coordinate.

(B) How does HOPS binding affect the force that the bound SNARE complex exerts on a fusion intermediate. To this end, we used simulation system 2 (see above) with the distance between the C-termini of Nyv1 and Vam3 as a reaction coordinate.

In protocol A, we pull two hydrophilic 'beads' (probes) through the stalk center in order to estimate the free energy required to open the fusion pore. Each 
probe is comprised of 8 clustered solvent beads. The "stalk thickness" is defined by the distance between the two probes. The rationale is to bring the system close to the nucleation barrier until that barrier can be crossed spontaneously within the simulation time scales. The work required to enforce nucleation provides an estimate of the height of the barrier. For more detailed information see our previous work ${ }^{32}$.

In protocol B, we study how HOPS binding alters the force that the C-termini of Nyv1 and Vam3 exert on the stalk intermediate. We rationalize such an effect from the relative change in work required to slightly pull the SNARE Ctermini (Nyv1 and Vam3) in closer proximity in the presence or absence of HOPS. Slightly implies that we only indent/squeeze the stalk such that the stalk will recover if no active pulling force is applied anymore (thermodynamically reversible).

To derive the associated free energies in both of these protocols, we applied umbrella simulation techniques $\left(K_{\text {force }}=1000 \mathrm{~kJ} \mathrm{~nm}^{-2} \mathrm{~mol}^{-1}\right)$ in combination with the weighted histogram method. We generated independent states along the reaction coordinate ( 50 for protocol A, 15 for protocol $B$ ) by performing a stirred molecular dynamics simulation over the entire reaction coordinate (pull rate: $-5 \times 10^{-5} \mathrm{~nm} / \mathrm{ps}$ ) in a pre-equilibrated system. Equilibrium was characterized by the pressure (bending work) and the resultant force on HOPS. For protocol A, a separate, independent stirred MD run must be performed for each different system (e.g., the attractive HOPS case) because the generated states will embed information about the nature of the barrier. The umbrella simulations were performed after the systems were equilibrated for $\sim 1.6$ us, i.e., the last snapshot was used for a stirred MD simulation, from which the different umbrella windows were generated. Equilibrium was characterized by the pressure (bending work) and the resultant force on HOPS. Each umbrella window was simulated over an effective time of $400-$ $600 \mathrm{~ns}$ to obtain overlapping distributions from which the total free energy profile was constructed. We discarded the first $40 \mathrm{~ns}$ of the simulation to ensure equilibration of the measured biased force. All of the free energy profiles and the error bars herein were obtained by using the Weighted 
Histogram Analysis Method (WHAM) in combination with the Bayesian bootstrapping method ${ }^{41}$. The bootstrapping method exploits the WHAM equations to reconstruct a large multiple of free energy profiles from resampled bootstrap histograms. The errors within the final free energy profile are estimated from the statistical fluctuations herein. Each re-sampled histogram of the biased force is reconstructed from the data which comprise the original histogram (an umbrella window) by random selection with replacement. This resampling procedure respects the (on the fly estimated) integrated autocorrelation time within the biasing force and adds an additional random weight to the histogram within the WHAM equations (Bayesian bootstrapping). An excellent detailed description of this procedure has been given $^{41}$.

\section{Bending energy and force}

The bending work is estimated from the total (equilibrium) force that the membrane exerts on the surface of HOPS after it adopts its shape of minimal free energy. The work $\left(W_{\text {eq }}\right)$ that HOPS must perform to bend the membrane is given by the relationship $W_{\text {eq }}=1 / 3 \Sigma f_{\text {eq,av }} \bullet r$, where $\Sigma f_{\text {eq,av }}$ is the total average force that acts on the surface of the HOPS sphere during growth from radius $r=0$ to $r=r$. Our continuum elastic calculations revealed that $\Sigma f_{e q}$ rather linearly increases with $r$. Therefore, we approximated $\Sigma f_{\text {eq,av }}$ from $r=0$ to $r=r$ by $1 / 2 \sum f_{\text {eq }}$ at $r=r$. This linear approximation enables (instantaneous) calculation of $W_{\text {eq }}$ by averaging over a single simulation (at $r=r$ ). For a purely repulsive sphere these relationships approximate the concomitant bending energy of the adopted membrane shape. We expect, however, to slightly systematically overestimate the bending energy (by a few $k_{B} T$ ) since the force on the HOPS sphere will in reality vanish slightly before $r=0(r \approx 0.5 \mathrm{~nm})$. This is due to the approximately $1.0 \mathrm{~nm}$ inter-membrane separation resulting from the presence of the stalk. Notable, HOPS bends the membrane by actively pressing against the membrane surface (indentation). This additional stress term (e.g., thinning of the membrane) is omitted within the Hamiltonian of the continuum model but is not expected to largely contribute to the surface free energy. The peristaltic force $\left(f_{d}\right)$ on HOPS (see Extended Data Fig. 9 ) is calculated from projecting the resultant force on HOPS (a 3-vector) on the vector connecting 
the center of HOPS with the stalk center. Here, the stalk center is defined as the geometrical center between the C-terminus of NYV1 and VAM3. Finally, the average values of bending work and $f_{d}$ are obtained by averaging over 1 $2 \mu$ s equilibrium simulations. The error in the average value is obtained by block averaging.

\section{Continuum model}

Continuum models were performed by minimizing the Helfrich hamiltonian within the de Monge representation $z(x, y)$ subjected to two constraints: (i) The inter-membrane constraint (a stalk or partly-assembled SNARE complex) and, (ii) a solid sphere (HOPS). The membrane was described by a discretized sheet consisting of 100 grid elements in the $y$, and 200 in the $x$ dimension. For reasons of symmetry we only model a quarter of the actual system and recover the full surface free energy by multiplication with a factor of 4 (for the projected area by a factor of 2). Reflecting boundary conditions were used in the $y$-dimension (the symmetry axis), periodic in the $x$-dimension. The actual membrane contact surface in vacuole fusion is of microscopic dimension ${ }^{42-44}$, indicating that a large multiple of constraints must be present and that the membrane is not 'free standing' even over large distances. All dimensions are based on the positions of the C4 lipid tail beads (the mid-plane of the membrane) within the MD simulations. Finally, HOPS embeds the SNARE complex non-symmetrically ${ }^{11}$ and will therefore induce torque. Torque (a three body force) does not occur when the distance (d) between the center of HOPS and the stalk (the two constraints) is used as a reaction coordinate but can be additionally derived/constructed by scaling the here-presented force $\left(f_{d}\right)$ with $a \sin (\theta)$ term.

The Hamiltonian for each surface element, $F_{\text {total, }}$ is given by $F_{\text {bending }}+F_{\text {constraint }}$ $+F_{\text {HOPS }}$, with $F_{\text {bending }}$ being the Helfrich bending energy, $F_{\text {constraint }}$ the energy of the inter-membrane constraint (a stalk, fusion pore or partly-assembled SNARE complex) and $\mathrm{F}_{\text {HOPS }}$ the energy of the HOPS' sphere. $F_{\text {bending }}$ is given by $2 \mathrm{KH}^{2}$ with $\mathrm{H}$ being the mean curvature, and $\mathrm{k}$ the bending modulus ( $24 \mathrm{k}_{\mathrm{B}} \mathrm{T}$, see citation ${ }^{9}$.). The constraint $F_{\text {constraint is modeled by a set of stiff springs }}$ which restraints the membrane at a height of $z_{\text {equ }}=2 \mathrm{~nm}$ and which imposes 
a circular 'stalk' region with a radius of $d_{\text {stalk }}=2 \mathrm{~nm}$. $F_{\text {constraint }}$ depends on the distance $d$ between a surface element and the center of the 'stalk' region, and the height of the membrane $z$. $F_{\text {constraint }}=0$ if $d>2 \mathrm{~nm}$ and $1 / 2 \mathrm{k}_{\text {stalk }}\left(\mathrm{z}-\mathrm{z}_{\mathrm{eq}}\right)^{2}(\mathrm{~d}-$ $d_{\text {stalk) }}$ otherwise, with $k_{\text {stalk }}$ being the force constant $\left(k_{\text {stalk }}=100 k_{B} T / n^{5}\right)$. Here, the term $\left(d-d_{\text {stalk }}\right)$ ensures a smooth (differentiable) transition of the 'stalk' region. HOPS was modeled as a sphere with a diameter $\mathrm{D}_{\mathrm{H}}$. The center of the sphere is located within the $\mathrm{x}, \mathrm{y}$ plane at $\mathrm{z}=0$. Surface elements overlapping with the HOPS sphere experience an harmonic repulsion, $F_{\text {HOPS }}=1 / 2 k_{\text {HOPS }}\left(2 r-D_{H}\right)^{2}$ if $2 r<D_{H}$ and $F_{\text {HOPS }}=0$ otherwise, with $r$ being the distance of a surface element from the center of the sphere. The force constant $\mathrm{k}_{\text {HOPS }}$ was chosen to be $100 \mathrm{kB} / \mathrm{nm}^{4}$. The example of a (moderately) attractive HOPS was modeled using $\mathrm{F}_{\text {HOPS }}=\mathrm{K}_{\text {HOPS }} \mathrm{d}^{2}\left(\mathrm{~d}^{2}-\mathrm{C}\right)$ with $\mathrm{C}=0.5 \mathrm{~nm}^{2}$, and $\mathrm{K}_{\text {HOPS }}=20 \mathrm{kB} / \mathrm{nm}^{6}$. Finally, the total surface free energy $\Sigma \mathrm{F}_{\text {totald }} \mathrm{A}$ was minimized using an over-damped deterministic minimization scheme. We emphasize that the values predicted by the continuum model are subject to simplification of the characteristics of the system (e.g. exact spatial dimensions, and the nature of the inter-membrane restraint). They also rely on the estimation of the bending modulus of the membrane.

\section{Code availability}

The molecular dynamics simulations were performed with a self-modified version of the open source software Gromacs-4.5.5. The implementation of the here-used HOPS potentials is described in detail in the supplement. The code is publically available under http://nlor. theorie. physik.unigoettingen.de/ hrissel/code/

\section{Data availability}

All original data will be made available by the authors upon request. 


\section{References for Methods}

33. Janke, C. et al. A versatile toolbox for PCR-based tagging of yeast genes: new fluorescent proteins, more markers and promoter substitution cassettes. Yeast 21, 947-962 (2004).

34. Longtine, M. S. et al. Additional modules for versatile and economical PCR-based gene deletion and modification in Saccharomyces cerevisiae. Yeast 14, 953-961 (1998).

35. Kuznetsova, I. M., Turoverov, K. K. \& Uversky, V. N. What macromolecular crowding can do to a protein. Int J Mol Sci 15, 2309023140 (2014).

36. Mage, M. G. in Immunochemical Techniques, Part A 70, 142-150 (Elsevier, 1980).

37. Hess, B., Kutzner, C., van der Spoel, D. \& Lindahl, E. GROMACS 4: Algorithms for Highly Efficient, Load-Balanced, and Scalable Molecular Simulation. J Chem Theory Comput 4, 435-447 (2008).

38. Monticelli, L. et al. The MARTINI Coarse-Grained Force Field: Extension to Proteins. J Chem Theory Comput 4, 819-834 (2008).

39. Marrink, S. J., Risselada, H. J., Yefimov, S., Tieleman, D. P. \& de Vries, A. H. The MARTINI force field: coarse grained model for biomolecular simulations. The journal of physical chemistry $B 111,7812-7824$ (2007).

40. Risselada, H. J., Kutzner, C. \& Grubmüller, H. Caught in the act: visualization of SNARE-mediated fusion events in molecular detail. Chembiochem 12, 1049-1055 (2011).

41. Hub, J. S., De Groot, B. L. \& van der Spoel, D. g_wham-A Free Weighted Histogram Analysis Implementation Including Robust Error and Autocorrelation Estimates. J Chem Theory Comput 6, 3713-3720 (2010).

42. Mayer, A. \& Wickner, W. Docking of yeast vacuoles is catalyzed by the Ras-like GTPase Ypt7p after symmetric priming by Sec18p (NSF). J Cell Biol 136, 307-317 (1997).

43. Wang, L., Merz, A. J., Collins, K. M. \& Wickner, W. Hierarchy of protein assembly at the vertex ring domain for yeast vacuole docking and 
fusion. J Cell Biol 160, 365-374 (2003).

44. Karunakaran, S., Sasser, T., Rajalekshmi, S. \& Fratti, R. A. SNAREs, HOPS, and regulatory lipids control the dynamics of vacuolar actin during homotypic fusion. J Cell Sci (2012). doi:10.1242/jcs.091900

45. Merz, A. J. \& Wickner, W. T. Resolution of organelle docking and fusion kinetics in a cell-free assay. 101, 11548-11553 (2004).

46. Lürick, A. et al. Multivalent Rab interactions determine tether-mediated membrane fusion. Mol. Biol. Cell mbc.E16-11-0764 (2016). doi:10.1091/mbc.E16-11-0764

47. Jackson, M. B. Minimum Membrane Bending Energies of Fusion Pores. J Membr Biol 231, 101-115 (2009).

48. Ryham, R. J., Ward, M. A. \& Cohen, F. S. Teardrop shapes minimize bending energy of fusion pores connecting planar bilayers. Phys. Rev. E 88, 693 (2013).

49. D'Agostino, M., Risselada, H. J. \& Mayer, A. Steric hindrance of SNARE transmembrane domain organization impairs the hemifusion-to-fusion transition. EMBO Rep 17, 1590-1608 (2016).

50. Pieren, M., Desfougères, Y., Michaillat, L., Schmidt, A. \& Mayer, A. Vacuolar SNARE protein transmembrane domains serve as nonspecific membrane anchors with unequal roles in lipid mixing. $J$. Biol. Chem. 290, 12821-12832 (2015) 


\section{Extended Data Figure Legends:}

\section{Extended Data Fig. 1: Reagents.}

a, HOPS is lacking on ypt7 $\Delta$ vacuoles. HOPS and Ypt7 content of total cell extracts and purified vacuoles from wildtype and ypt7 $\Delta$ mutants in BJ3505 and DKY6281 cells, representing the background strains used for the content mixing assay. b, Purified HOPS, HOPS subcomplexes and CORVET. The complexes used for the in vitro experiments were analyzed by SDS-PAGE and Coomassie staining. The gel represents the preparations, which followed published routine procedure, used for the experiments in Fig. 1 and Extended Data Figs. 3 and 4. c, Production of $F_{a b}$ fragments from polyclonal antibodies to Vam3 and Nyv1. Schematic view of papain cleavage sites for $F_{a b}$ fragment generation on the left. Affinity-purified antibodies and $F_{a b}$ fragments extracted after papain digestion were analyzed by non-reducing SDS-PAGE and Coomassie staining. The gel shows the preparation used in Fig. 2. d, Expression of FKBP and FRB fusion proteins. Total cell extracts were prepared from $0.1 \mathrm{OD}_{600 \mathrm{~nm}}$ units of logarithmic cultures of yeast strains expressing Vam7-2xFKBP12 and/or Pfk1-FRB-GFP. Proteins were analyzed by SDS-PAGE and Western Blotting against Vam7, Vam3 and GFP. e, Same as in d, but for cells expressing Vam7-LL-2xFKBP12, containing the 35 aa linker (LL). For a and c-e, similar results were obtained from two independent preparations.

\section{Extended Data Fig. 2: Kinetics and efficiency of in vitro vacuole fusion, measured by content mixing.}

Vacuoles are prepared from two different strains, which contain either the soluble 45 kDa maturase Pep4 (contained in DKY6281) or the pro-alkaline phosphatase p-ALP (contained in BJ3505). Formation of a sufficiently large fusion pore allows Pep4 to transfer into the p-ALP containing fusion partner, leading to proteolytic cleavage of its pro-sequence and activation of the enzyme (m-ALP). This activity is measured as a readout for fusion. Note that 
proteolytic maturation of p-ALP is fast and not limiting for the development of the content mixing signal ${ }^{45}$. Standard fusion reactions have been started. At the indicated time points, aliquots were withdrawn and set on ice. a, At the end of the 60 min period, m-ALP activity was determined for all samples. Means \pm s.d. are shown for $n=3$ biologically independent experiments. $\mathbf{b}$, Aliquots from one of the experiments in (a) were TCA-precipitated and analyzed by SDS-PAGE and Western blotting against ALP and Vam3. Signals were detected on a LICOR infrared scanner (left) and quantified (right) as the ratio $m-A L P ~ / ~(m-A L P+p-A L P)$. Vam3 has been decorated as a loading control. Note that after one round of fusion, only a maximum of $50 \%$ of $p$-ALP can be matured, because half of the fusion events in the suspension will occur between like vacuoles (i.e. Pep4/Pep4 or p-ALP/p-ALP) and will not produce a signal.

Extended Data Fig. 3: HOPS and CORVET complexes stimulate fusion to similar degrees.

Two-stage fusion reactions with ypt7 $\Delta$ vacuoles were run as in Fig. $1 \mathrm{~g}$, in the absence of ATP. rVam7 had been added in the first phase of the incubation, $0.4 \mu \mathrm{M}$ HOPS subcomplexes or $0.4 \mu \mathrm{M}$ CORVET only in the second. Half of the samples received an addition of $6 \%$ of Ficoll 400 in the second incubation, an agent mimicking molecular crowding ${ }^{35}$. At the end of the 75 min incubation period, content mixing was assayed. Fusion activity of a standard wildtype reaction performed in the presence of ATP served as $100 \%$ reference. Means \pm s.d. are shown for $n=3$ biologically independent experiments.

\section{Extended Data Fig. 4: ypt7 $\Delta$ vacuoles require both Vam7 and HOPS for content mixing.}

Two-stage fusion reactions were run as in Fig. $1 \mathrm{~g}$ in the presence or absence of ATP. rVam7 had been added in the first $\left(1^{\circ}\right)$ phase of the incubation, HOPS only in the second $\left(\mathrm{II}^{\circ}\right)$. At the end of the $75 \mathrm{~min}$ incubation period, content mixing was assayed. Means \pm s.d. are shown for $n=3$ biologically independent experiments. 
Extended Data Fig. 5: A molecular crowding agent cannot stimulate fusion in the absence of bulky SNARE ligands.

Two-stage fusion reactions with ypt7 $\Delta$ vacuoles were run as in Fig. $2 \mathrm{~b}$, in the absence or presence of ATP. rVam7 had been added in the first phase of the incubation where indicated, antibodies and various concentrations of the crowding agent Ficoll 400 only in the second. At the end of the $75 \mathrm{~min}$ incubation period, content mixing was assayed. Fusion activity of a wildtype reaction performed in the presence of ATP served as $100 \%$ reference. Means \pm s.d. are shown for $n=3$ biologically independent experiments.

Extended Data Fig. 6: Effect of rapamycin-induced dimerization on invivo vacuole fusion using the small fusion protein FRB-GFP.

a, Schematic view of rapamycin-induced FKBP12/FRB-tagged protein dimerization between Vam7-2xFKBP12 and FRB-GFP. b, Logarithmically growing cells, expressing tagged Vam7-2xFKBP12, were stained with the vacuole tracer FM4-64 and analyzed by spinning disc microscopy before and $10 \mathrm{~min}$ after the addition of $10 \mu \mathrm{M}$ rapamycin. Scale bar: $5 \mu \mathrm{m}$. Similar results were obtained in 3 biologically independent repetitions.

\section{Extended Data Fig. 7: Fusion can be prematurely triggered by protein} recruitment after osmotically induced vacuole fragmentation.

a, Logarithmically growing cells, carrying Vam7-2xFKBP12 and Pfk1-FRBGFP as indicated, were stained with the vacuole tracer FM4-64. Vacuole fission was induced by adding $0.5 \mathrm{M} \mathrm{NaCl}$. Cells were analyzed by spinning disc microscopy before and 10 and 60 min after salt addition. The cells were grouped into three categories according to the number of vacuoles visible per cell. 100 cells were analyzed per sample. Values represent the means and s.d. from $\mathrm{n}=3$ biologically independent experiments. Scale bar: $5 \mu \mathrm{m}$. b, As in a, but $10 \mu \mathrm{M}$ rapamycin was added before the salt shock. c, As in b, but with cells expressing non-tagged Vam7. 
Extended Data Fig. 8: Effect of HOPS on the free energy barrier of fusion pore formation. This plot complements Fig. 4 in the main manuscript.

a. The free energy barrier of fusion pore opening is derived for a simulated system consisting of 3 SNARE complexes (panels on the right side) and a POPC membrane that contains 40\% POPE (colored orange). To this aim, we pull two hydrophilic probes (colored purple) towards the center of the stalk and estimate the work $(\Delta G)$ as a function of probe - probe distance (the stalk thickness $)^{32}$. The arrows in the free energy profile indicate the nucleation barrier for the fusion pore. Beyond this stage, subsequent pore opening proceeds in the absence of additional work (the plateau region). Tethering proteins such as HOPS are attracted to the membrane through Rab-GTPases or direct lipid interaction ${ }^{10,46}$. An attractive 'HOPS' surface (green line) conserves the lowered nucleation barrier, even when the surface attractions fully compensate the membrane bending energy (no net bending work; Extended Data Fig. 9). Error bars are calculated via Bayesian resampling of 50 overlapping WHAM histograms [43]. Each parental WHAM histogram is comprised of $>30000$ data points (autocorrelation up to $\sim 1500$ data points). $\mathbf{b}$, Pore formation in the absence of HOPS. A defect is frequently formed in the vicinity of the SNARE TMDs (black arrow), illustrating the presence of a high stress (the defect likely decreases the bending stress). Fusion pore formation is associated with a sudden reduction of the sharp curvature near the stalk's circumference (dashed lines). Fusion pores tend to adopt a teardrop shape 47,48. c, Fusion pore formation in the presence of HOPS. The pre-existing teardrop membrane shape imposed by HOPS likely provides a geometrical and therefore an energetic advantage for pore formation. $\mathbf{d}$, Setup where we artificially enforced formation of a leakage pore/defect in the direct vicinity of the stalk (the rationale behind this has been explained ${ }^{32}$ ). The induced defect (Between 3.2 and $1.8 \mathrm{~nm}$ the probe pierced through the membrane) instantaneously recovers. This suggests that the stress that HOPS imposes on the fusion site does not poise fusion to become leaky. 


\section{Extended Data Fig. 9: Detailed analysis of HOPS-mediated membrane}

bending in the presence of an inter-membrane restraint.

a, Simulation snapshot illustrating the geometry of the system. Shown is the central plane of the membrane (the lipid tail ends), the stalk, and HOPS. The SNARE complex present in the simulation setup is not illustrated. This setup serves as a motivation for the elastic continuum model. b, Bending work required to place HOPS at the (hemi-)fusion site and peristaltic force experienced by HOPS. Simulations were run to measure the work required to place HOPS-like spheres of 10-14 nm diameter at the site of hemifusion or at a fusion pore (FP). HOPS could be detached from the SNARE complex by a long spacer (link.). The influence of a SNARE complex with an unstructured, non-helical juxta-membrane region (unstr.) and of a HOPS mimic that was attractive to the membrane surface (attr.) was also analyzed. The lower panel shows averages obtained from the simulations. $f_{d}$ is the (peristaltic) force that pushes HOPS away from the inter-membrane restraint (e.g., a stalk, fusion pore, or trans-SNARE complex). Note that surface attractions or Rab-GTPase interactions of HOPS (modeling the tethering of membranes) ${ }^{10,46}$ can yield a negative value of the average work required to bend the membrane (bending occurs spontaneously). Fusion pore formation reduces the required bending work - it moves HOPS away from the restraint because of additional SNARE association up into the TMD region. The errors in the averages are derived from block averaging over $>10000$ data points until the error becomes independent of block size (autocorrelation up to $\sim 300$ data points). The errors in the averages are derived from block averaging over $>10^{\prime} 000$ data points until the error becomes independent of block size (autocorrelation up to $~ 300$ data points). c, Elastic continuum model. The coordinate system is based on the snapshot of the molecular dynamics simulation shown in panel a.

Because of symmetry along the xy-plane and xz-plane, we only model a quarter of the original system. The cartoon illustrates the shape of minimal free energy for a membrane (modeled by a single sheet), subjected to two constraints: (i) A local constraint on the position (height) of the membrane illustrated by the black arrow at $z=2 \mathrm{~nm}$. This mimics the inter-membrane constraint (stalk, fusion pore or partly-assembled SNARE complex), (ii) The presence of a hemisphere. This mimics HOPS. The color code illustrates the 
height of the membrane (the z-axis) relative to the two constraints. $d$, Prediction of bending energies by the elastic continuum model. The bending energy is shown as a function of the size and distance of HOPS to the intermembrane restraint. Upper panel: Bending energy decreases steeply when HOPS moves away from the restraint. The predicted values are about a factor of two lower than the "bending work" predicted by the simulations (see Methods). Middle panel: the corresponding peristaltic force $f_{d}$ on HOPS (the derivative of bending energy). At short distances, $f_{d}$ becomes substantial (tens of $\mathrm{pN}$ ). Note that making the surface of HOPS moderately attractive to the membrane affects $f_{d}$ only weakly, i.e. it does not result in an attraction towards the 'stalk'. Lowest panel: The relative reduction of membrane area as a result of HOPS-induced membrane bending. This property reflects the tension that HOPS induces by curving the membrane near the contact zone. In contrast to bending energy and force, tension only weakly depends on the distance $(d)$ to the restraint.

\section{Extended Data Fig. 10: Effect of HOPS on the force exerted by a single SNARE complex.}

a, One way of rationalizing the acceleration of fusion pore formation by a SNARE complex is to consider it as a mechanical device that exerts force on the luminal leaflets through its TMDs, thereby compressing the stalk. This can happen through a peristaltic force that pulls the SNARE complex away from the stalk, or through the elastic bending of the SNAREs. This latter mode of force transmission requires the juxta-membrane regions, which connect the coiled-coil domains of the SNAREs to their TMDs, to be structured and rigid. The compressing force that the SNARE complex exerts on the stalk can be rationalized from the apparent work (free energy) that one needs to perform in order to force the luminal C-termini of Vam3 and Nyv1 in closer proximity. We estimated how HOPS binding affects the force that the C-termini of the SNAREs Vam3 and Nyv1 exert on the stalk. b. The work required to slightly indent the stalk in the presence of repulsive or attractive HOPS-spheres of different diameter has been determined. It is shown relative to the situation without the sphere. Error bars are calculated via Bayesian resampling of 15 
overlapping WHAM histograms ${ }^{41}$. Each parental WHAM histogram is comprised of $>30^{\prime} 000$ data points (autocorrelation up to $\sim 1200$ data points). The lines shown result from fitting a power expansion (up to the 4th power) through the average of each data point. Error bars are calculated via Bayesian resampling of 15 overlapping WHAM histograms [43]. Each parental WHAM histogram is comprised of $>30$ '000 data points (autocorrelation up to $\sim 1200$ data points). The lines shown result from fitting a power expansion (up to the 4 th power) through the average of each data point. c, The corresponding forces on the SNARE TMDs were derived from this work. Apparent gains in the force exerted by the SNARE C-termini (left panel) are shown as a function of their distance in the hemifusion structure. HOPS binding can double or triple the magnitude of the apparent force $(10-20 \mathrm{pN})$ that a SNARE complex exerts on a stalk ${ }^{49}$. The gain dissipates, however, as zipping of the SNARE TMDs progresses and their C-termini approach each other. d, Snapshots of three special scenarios. Highest panel: The HOPS sphere is placed at a distal location with respect to the stalk (e.g., via attachment with a flexible linker). This abolishes the force gain. Middle panel: A sphere that favorably attracts (and bends) the membrane. This conserves the force gain. Lowest panel: Unstructured, flexible SNARE juxta-membrane regions partially disrupt the mechanical coupling between the coiled-coil domains and the TMDs. They decrease the apparent gain in SNARE pull force induced by HOPS. Structured ( $\alpha$-helical) SNARE juxta-membrane regions result in a high initial force gain which gradually reduces. In contrast, unstructured, flexible juxta-membrane regions, which impair vacuole fusion ${ }^{50}$, result in a near-constant force gain of only about $8 \mathrm{pN}$. Both cases converge to similar force values when the C-termini of Vam3 and Nyv1 come in closer proximity. Since the SNARE complex is unable to exert bending force on the membrane when the connection between its transmembrane anchors and the SNARE domains is completely flexible, we relate the remaining gain to an effective 'softening' of the stalk because of the induced membrane curvature and to the peristaltic force generated by the interaction of the HOPS sphere with the SNAREs. 
a

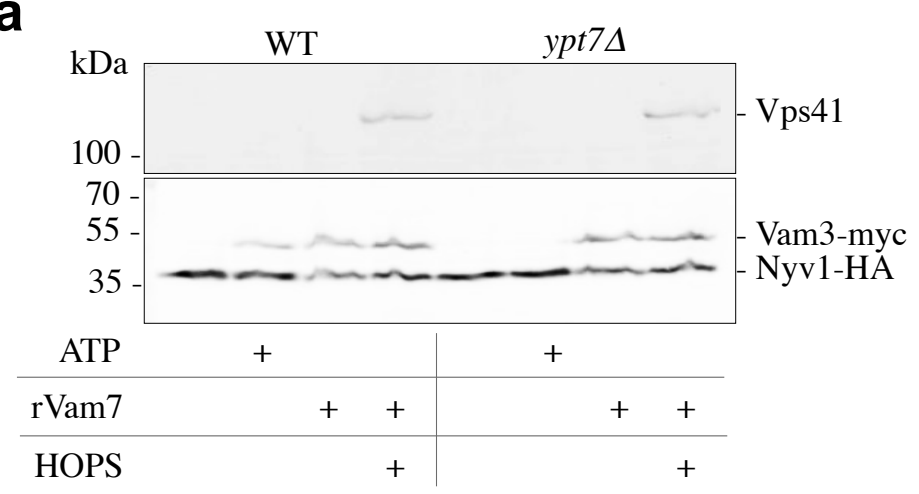

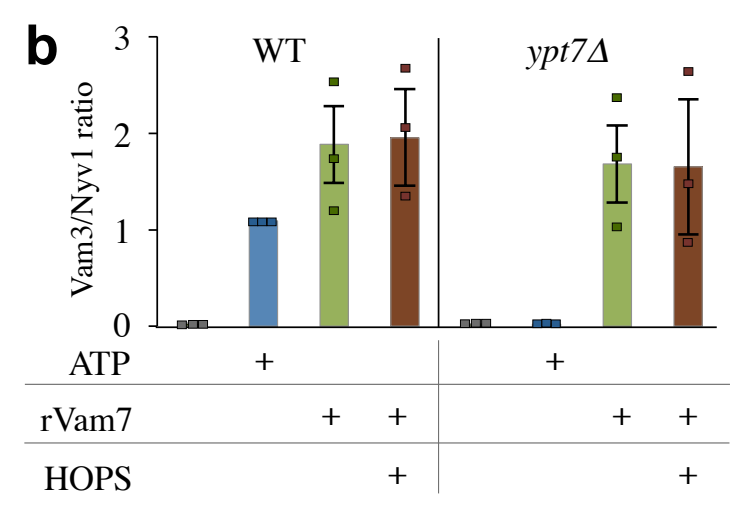
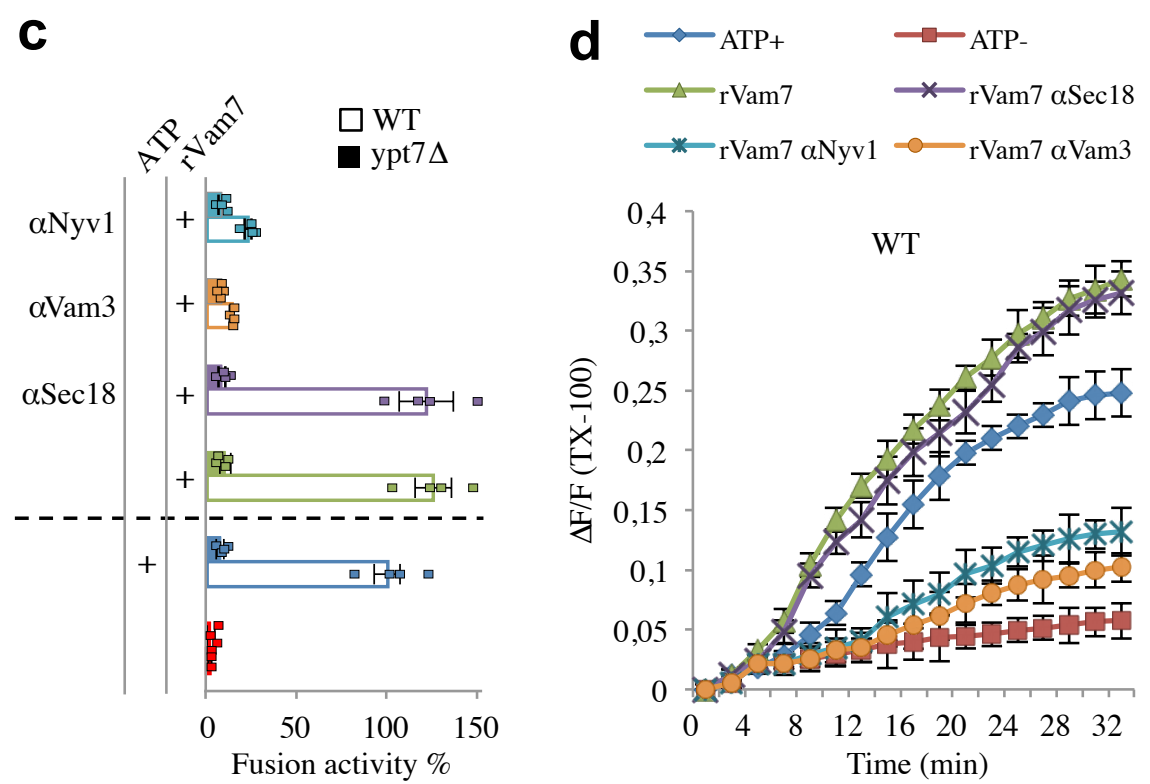

$\begin{array}{ll}\longrightarrow \text { ATP+ } & \longrightarrow \text { ATP- } \\ \rightarrow \text { rVam7 } & \rightarrow \text { rVam7 } \alpha \text { Sec18 } \\ \rightarrow-\text { rVam7 } \alpha \text { Nyv1 } & - \text { rVam7 } \alpha \operatorname{Vam} 3\end{array}$
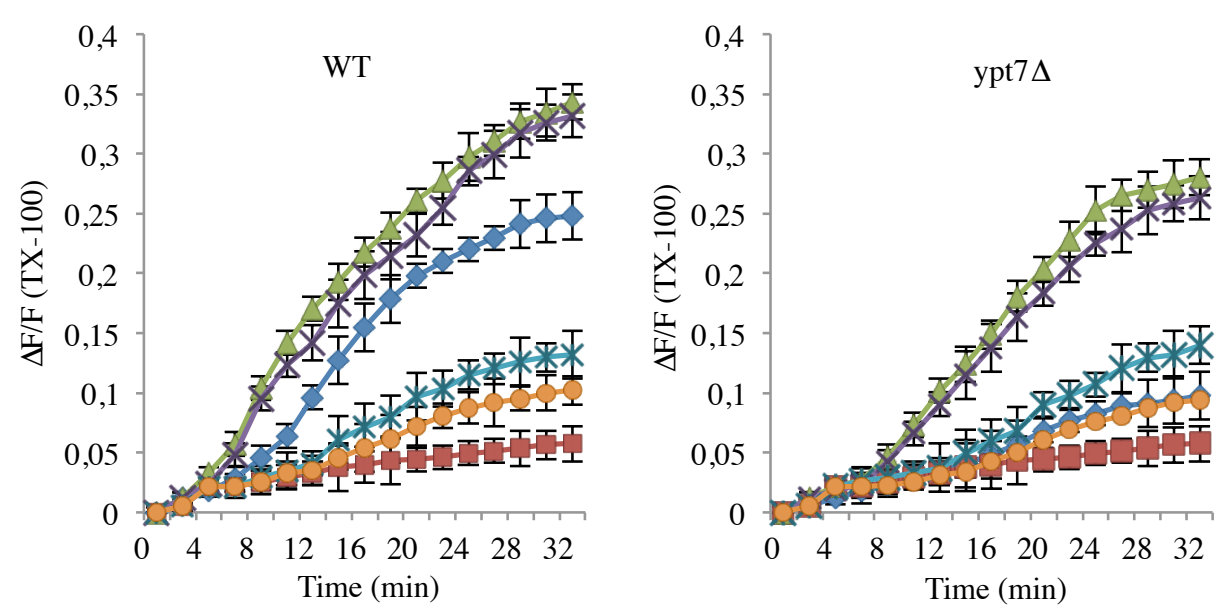

f

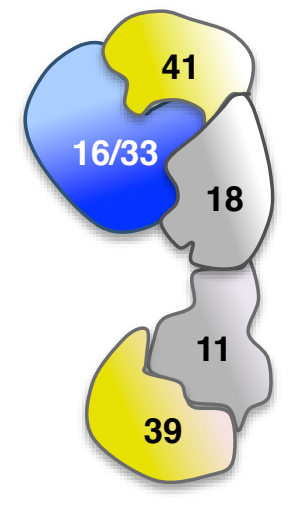

g

Vacuoles +/- rVam7 or $\alpha$ Vam3

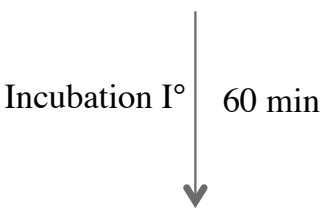

+/- HOPS subunits

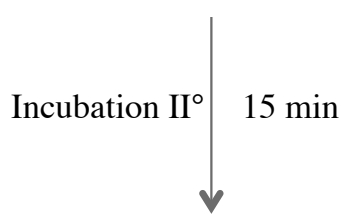

Measure content mixing

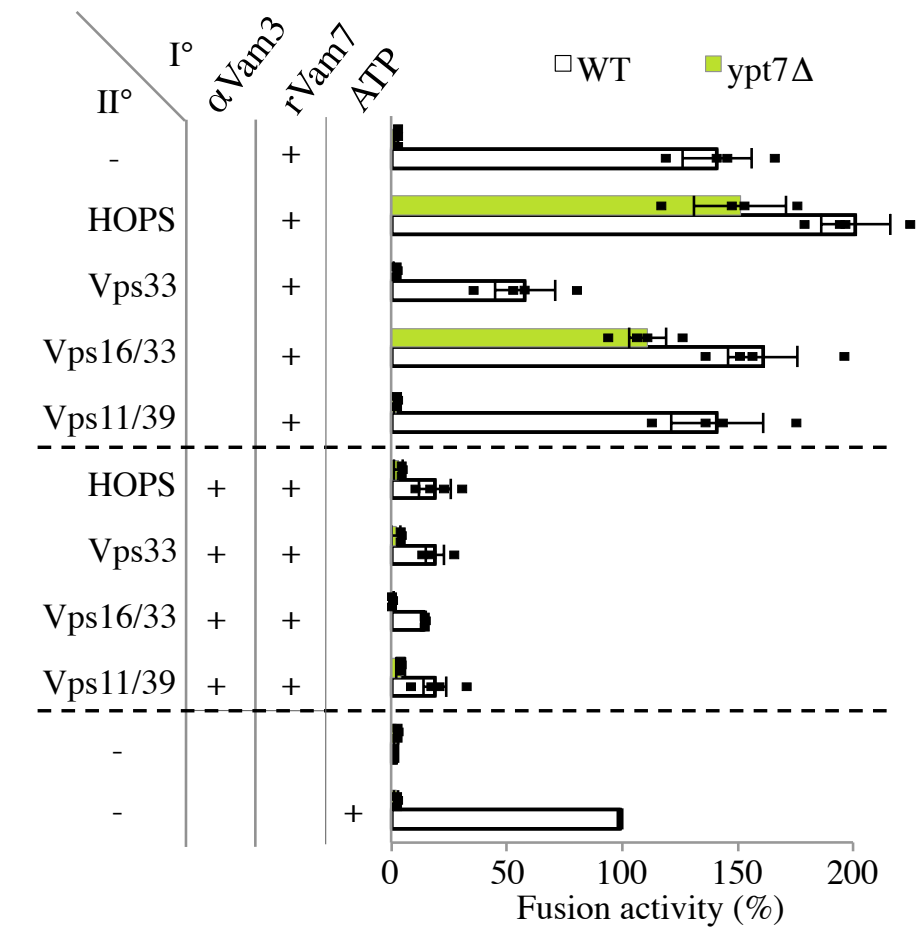


Fig. 2

a

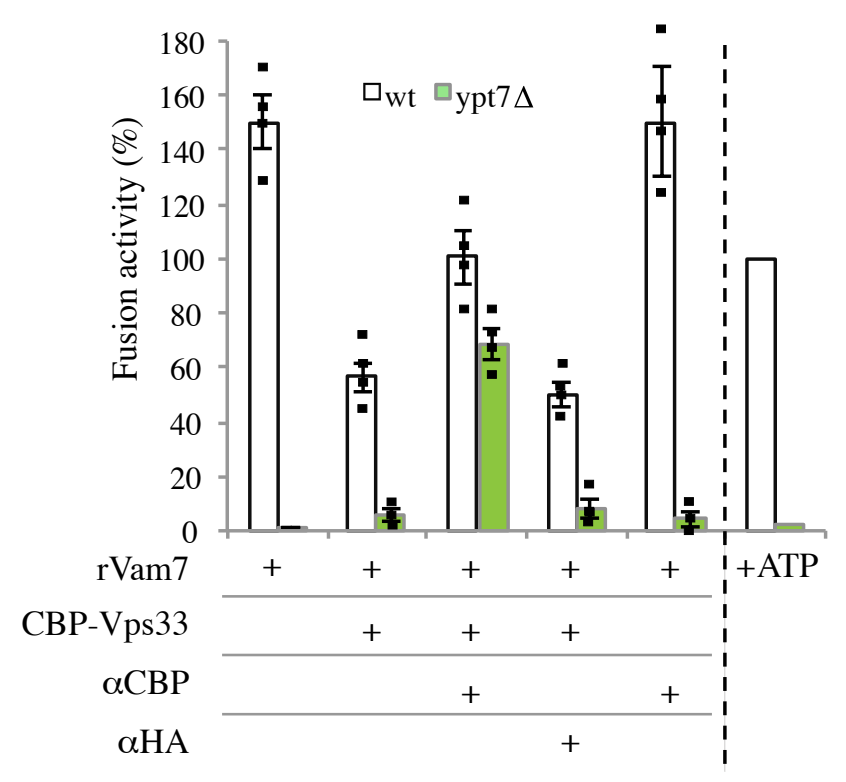

b

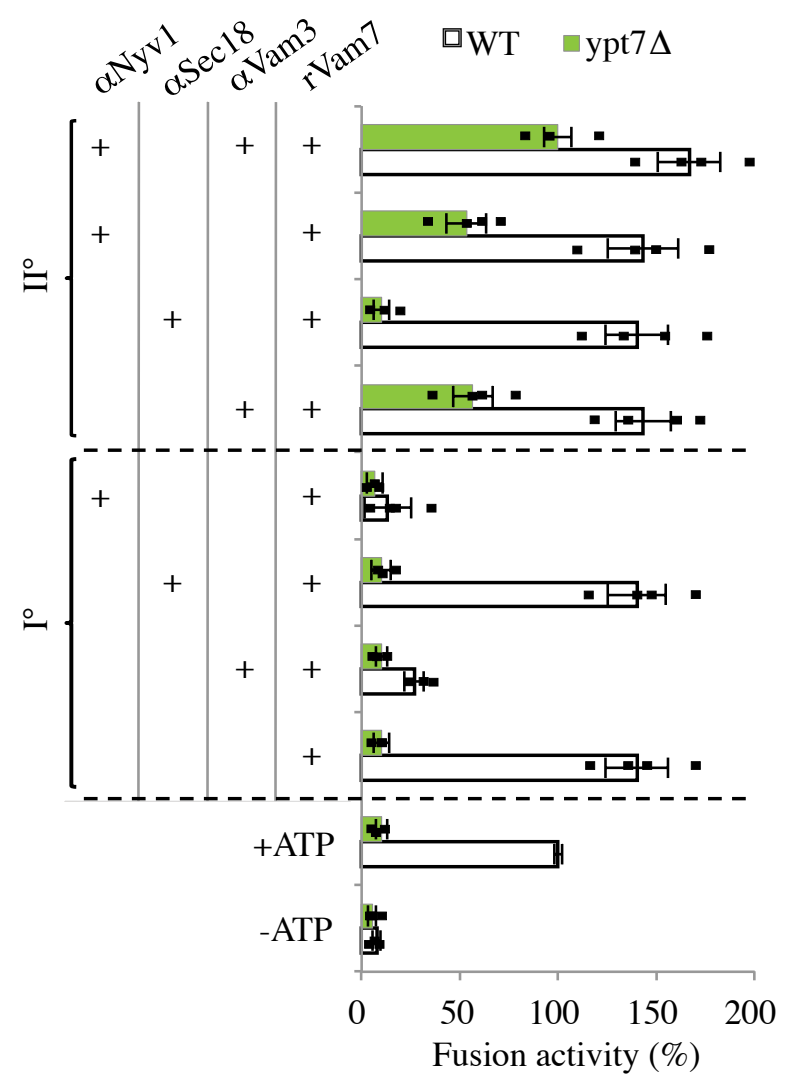

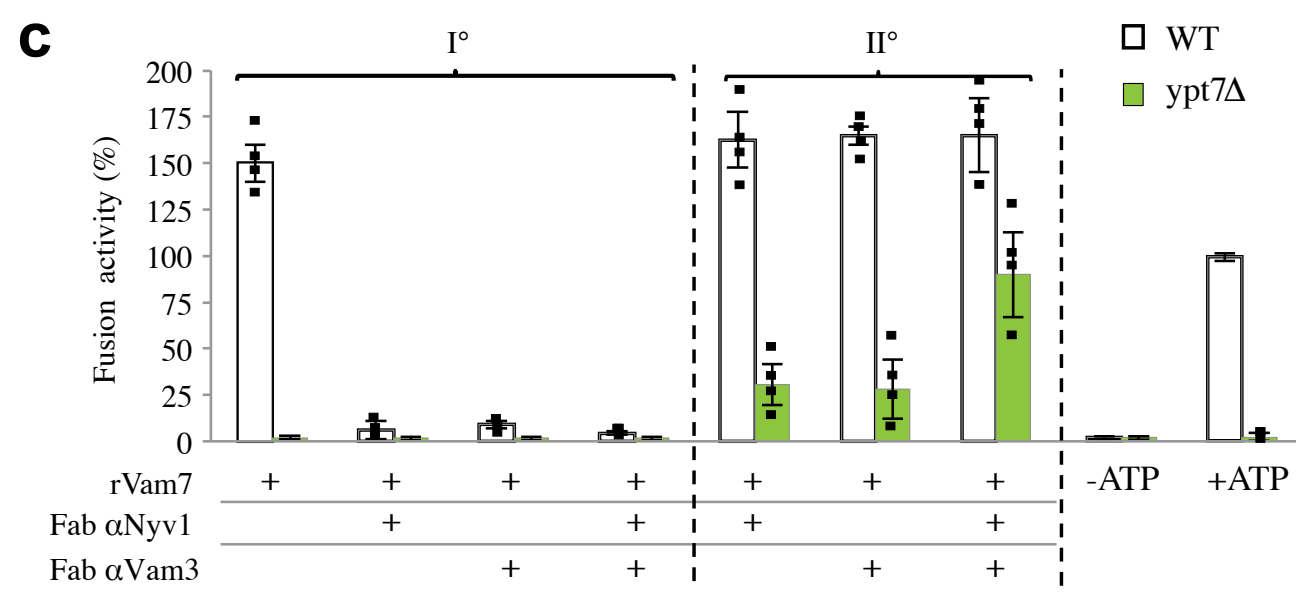

d
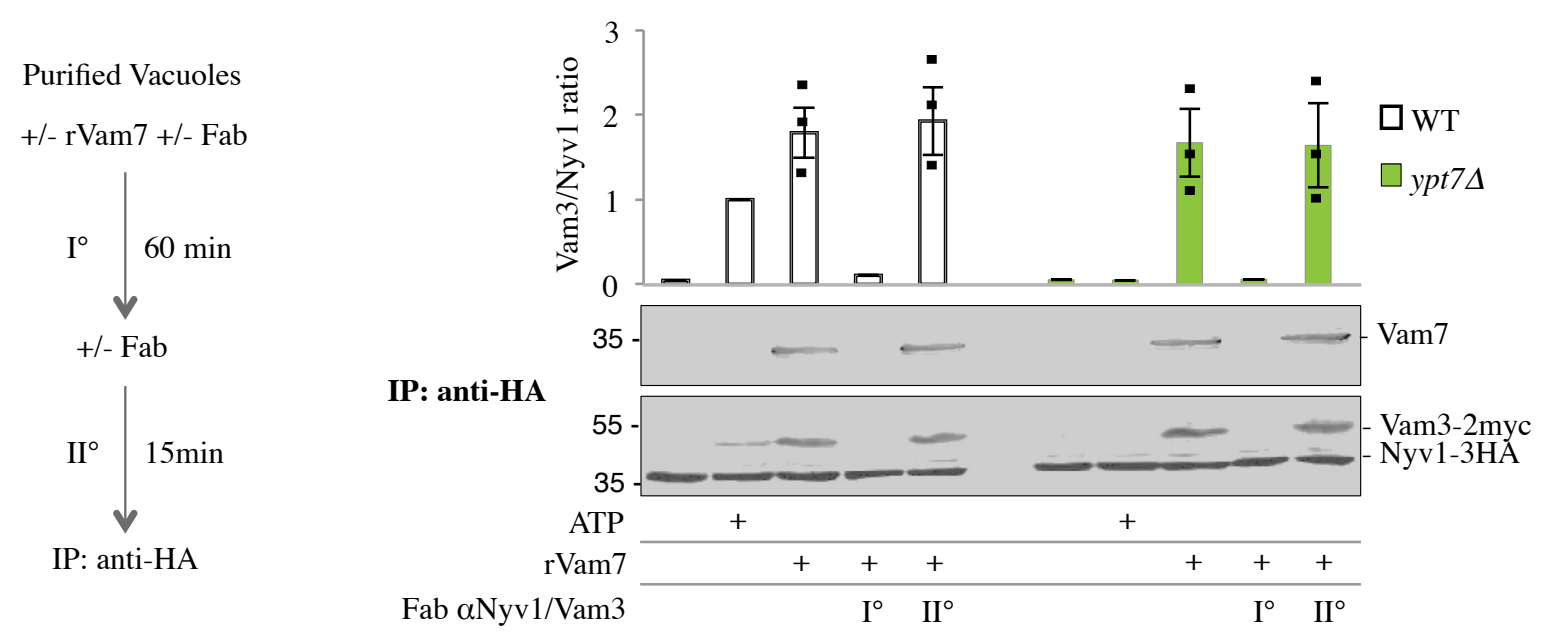

Fab aNyv1/Vam3 


\section{Fig. 3}

a

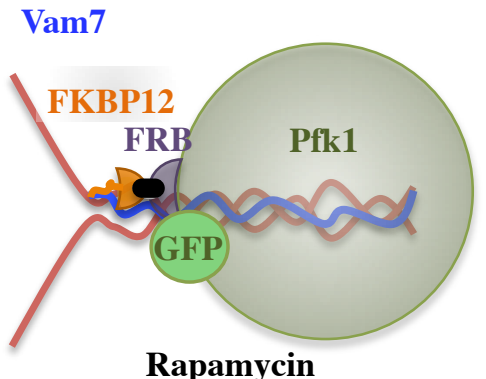

b
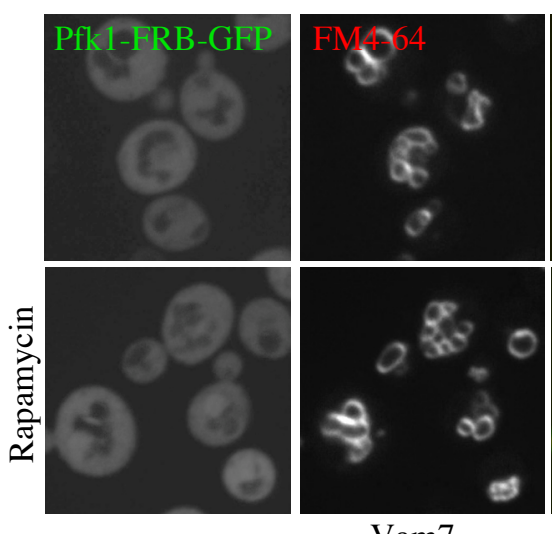

Vam7

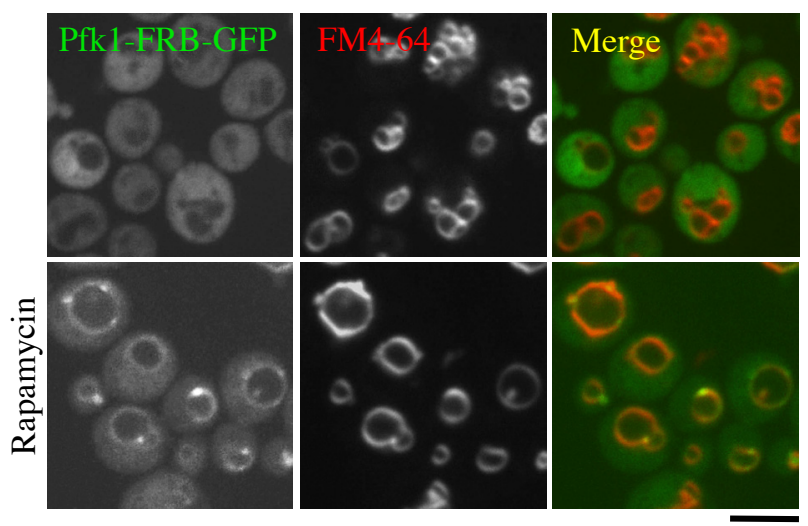

Vam7-2xFKBP12

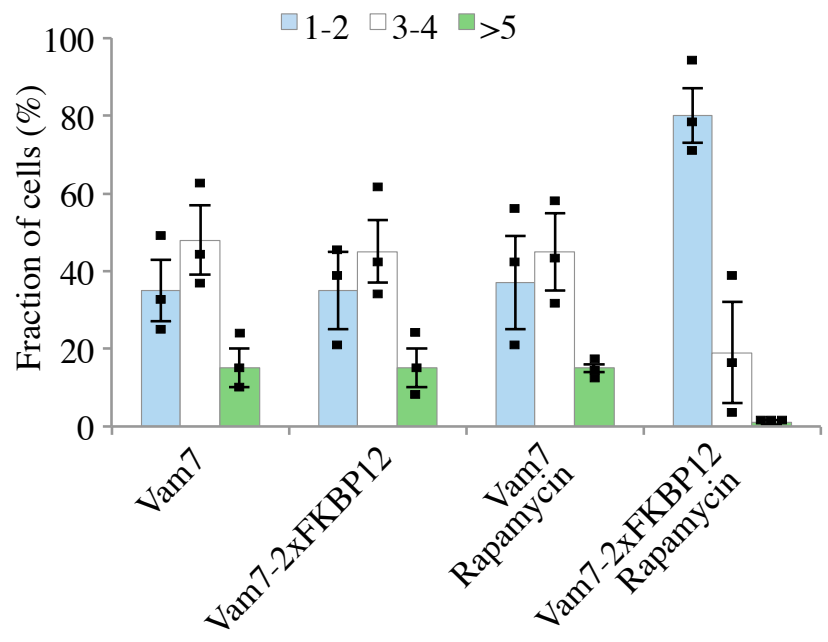

Vam7 long linker LL (35aa)

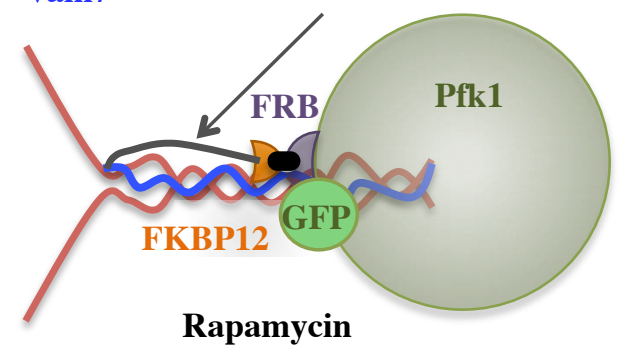

C

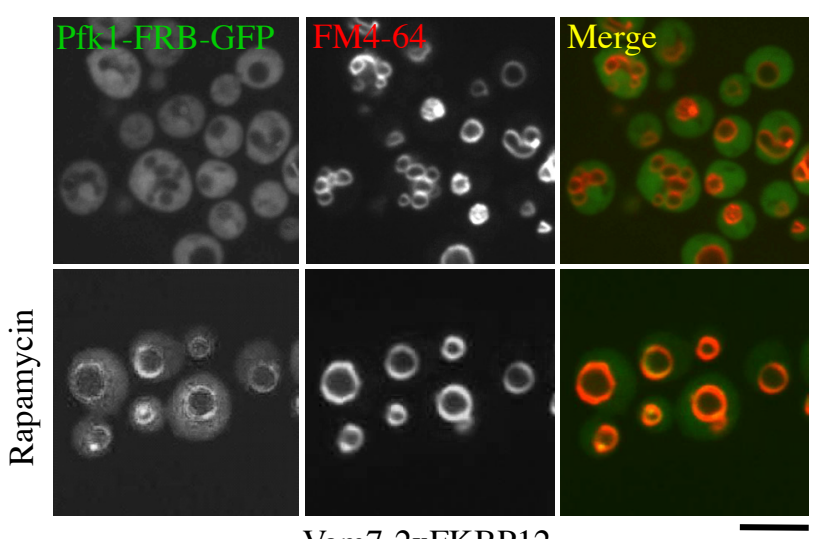

Vam7-2xFKBP12
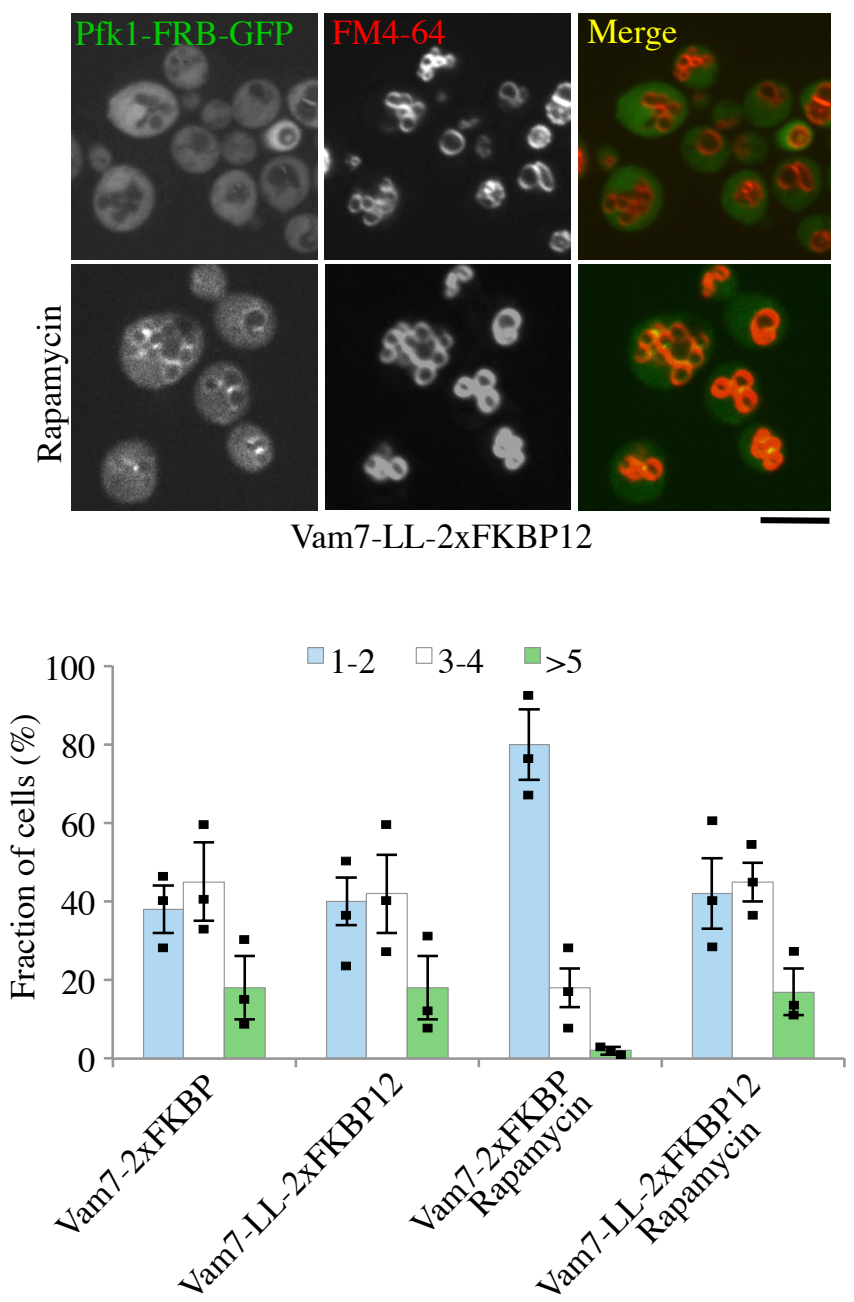


\section{lipid}

\section{SNARE complex}

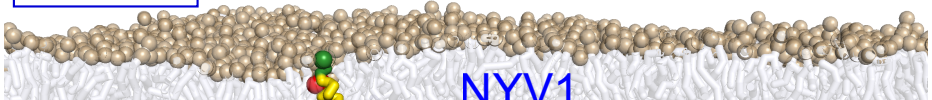

NYV1

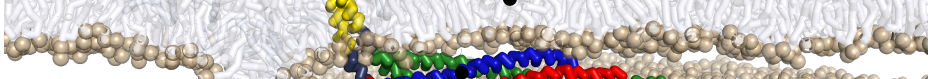

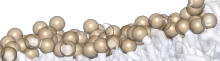

20 no HOPS (reference)

nhicknes
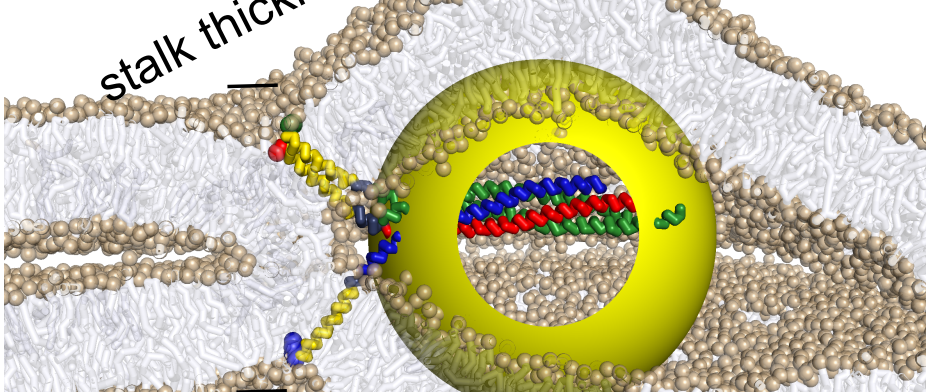

$14 \mathrm{~nm} \mathrm{HOPS}$ (stalk)

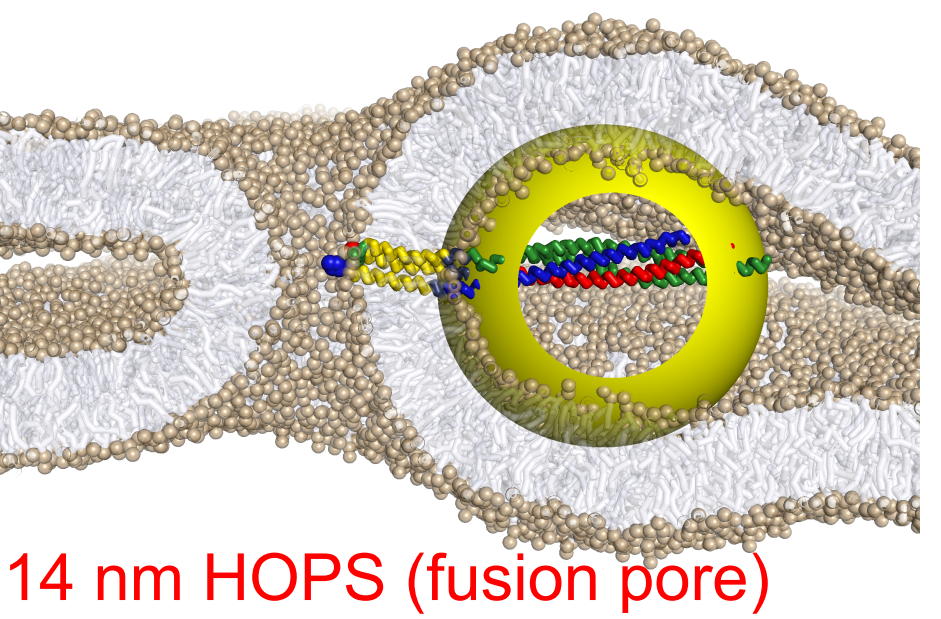

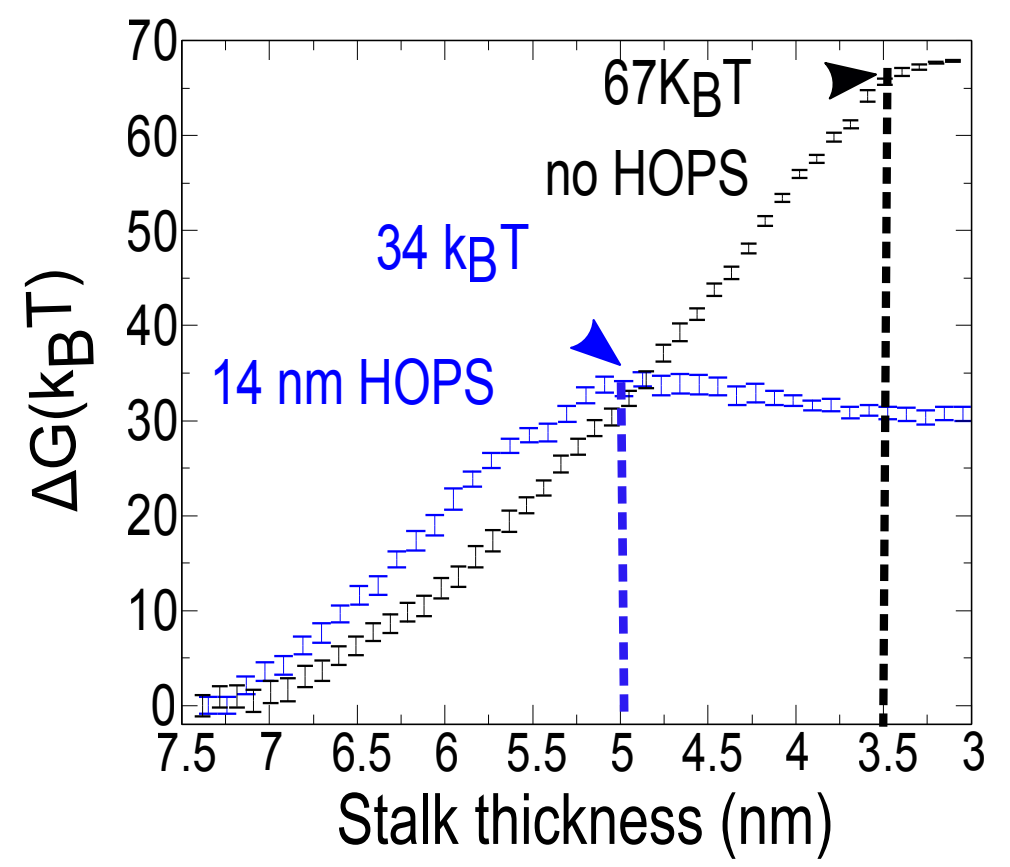

C

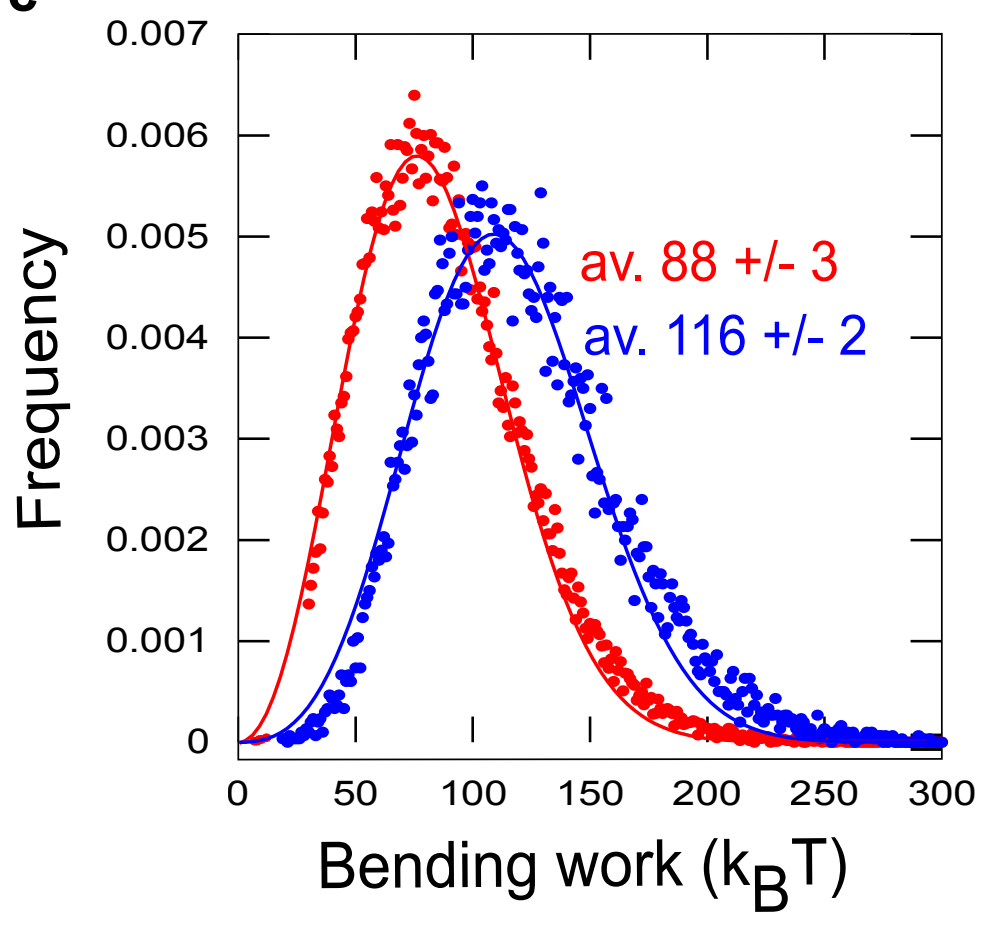




\section{Extended Data Figure 1}

a

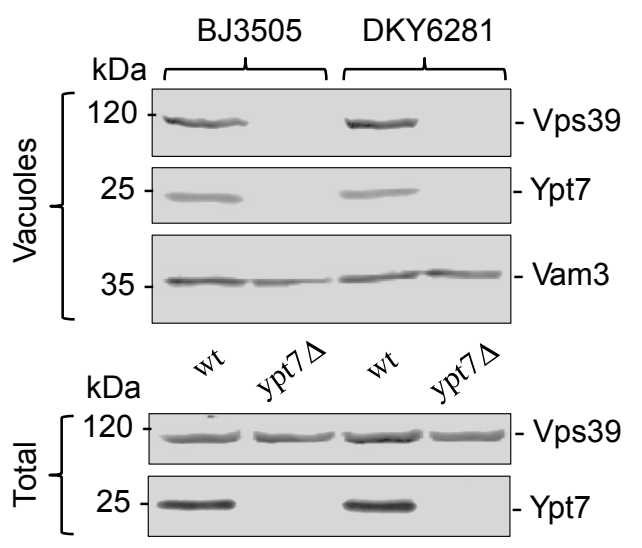

b

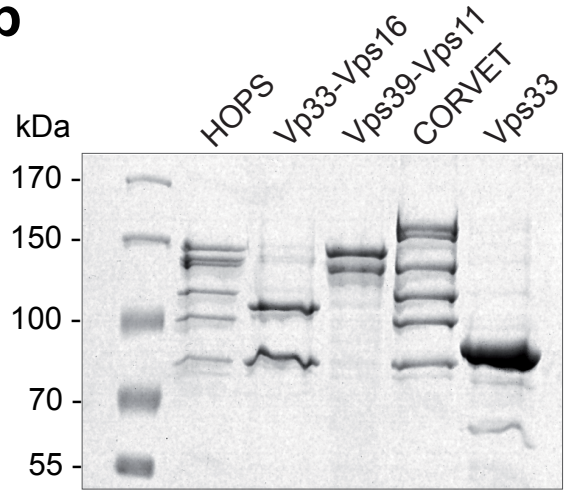

C

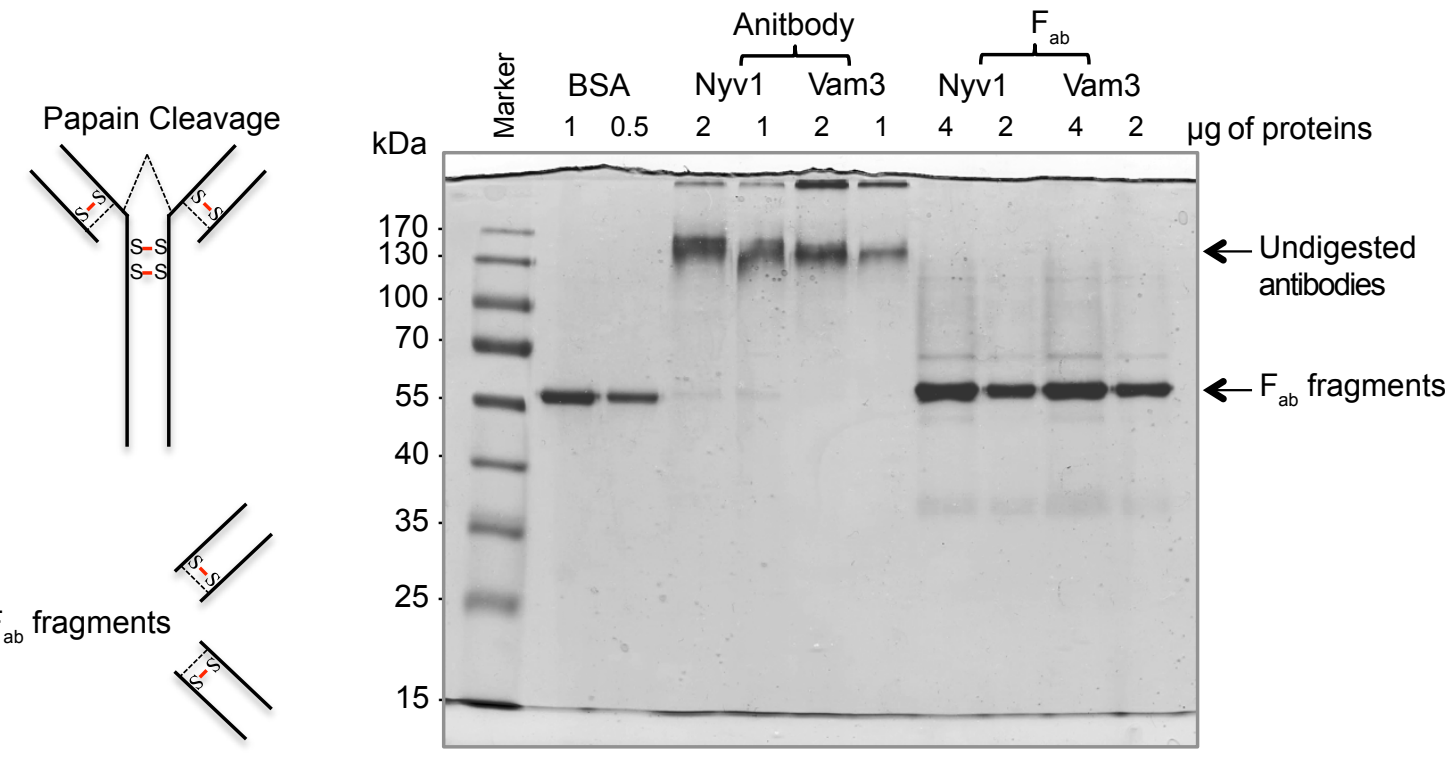

d

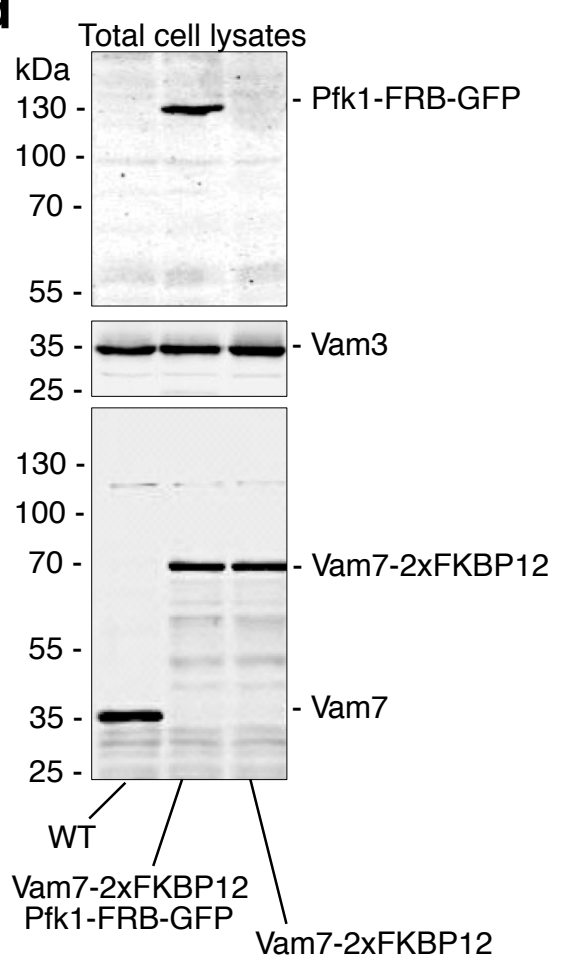

e

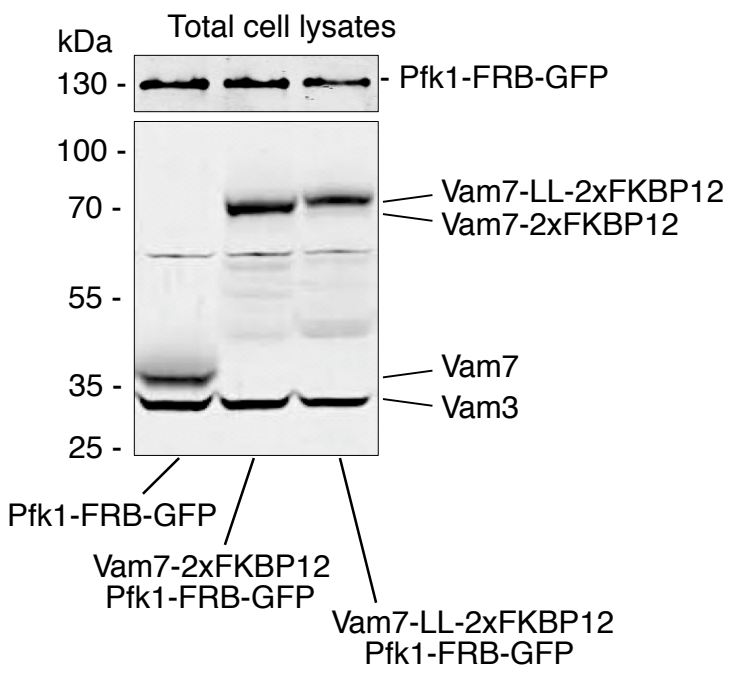




\section{Extended Data Figure 2}
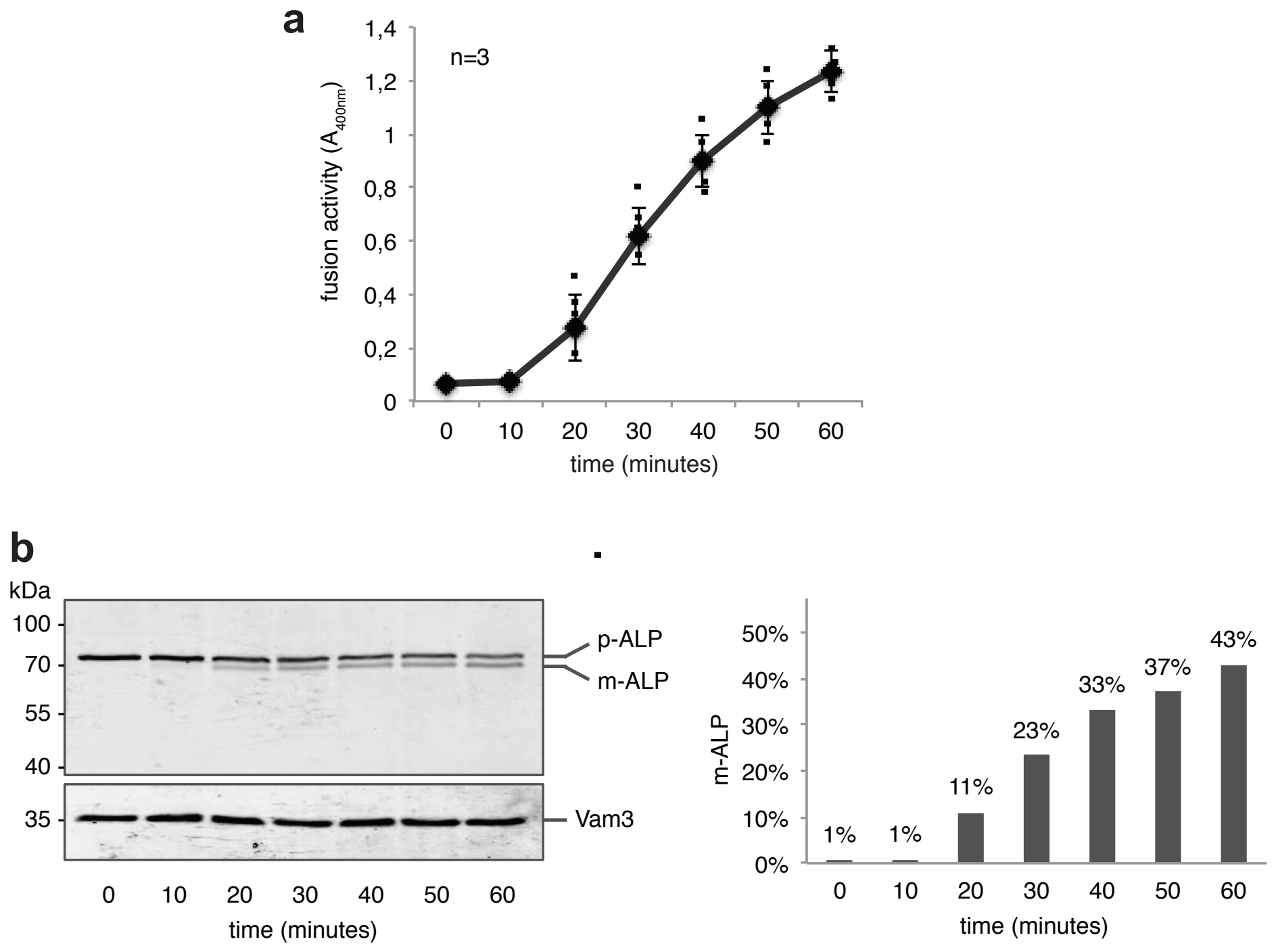


\section{Extended Data Figure 3}

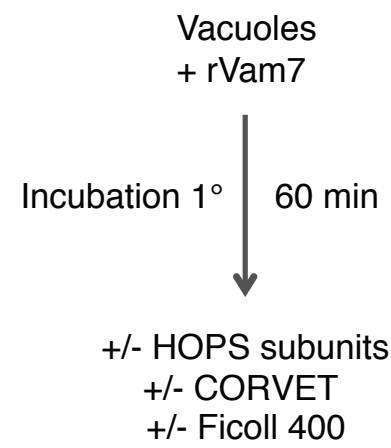

Incubation $\left.2^{\circ}\right\rfloor 15$ min

Measure

content mixing

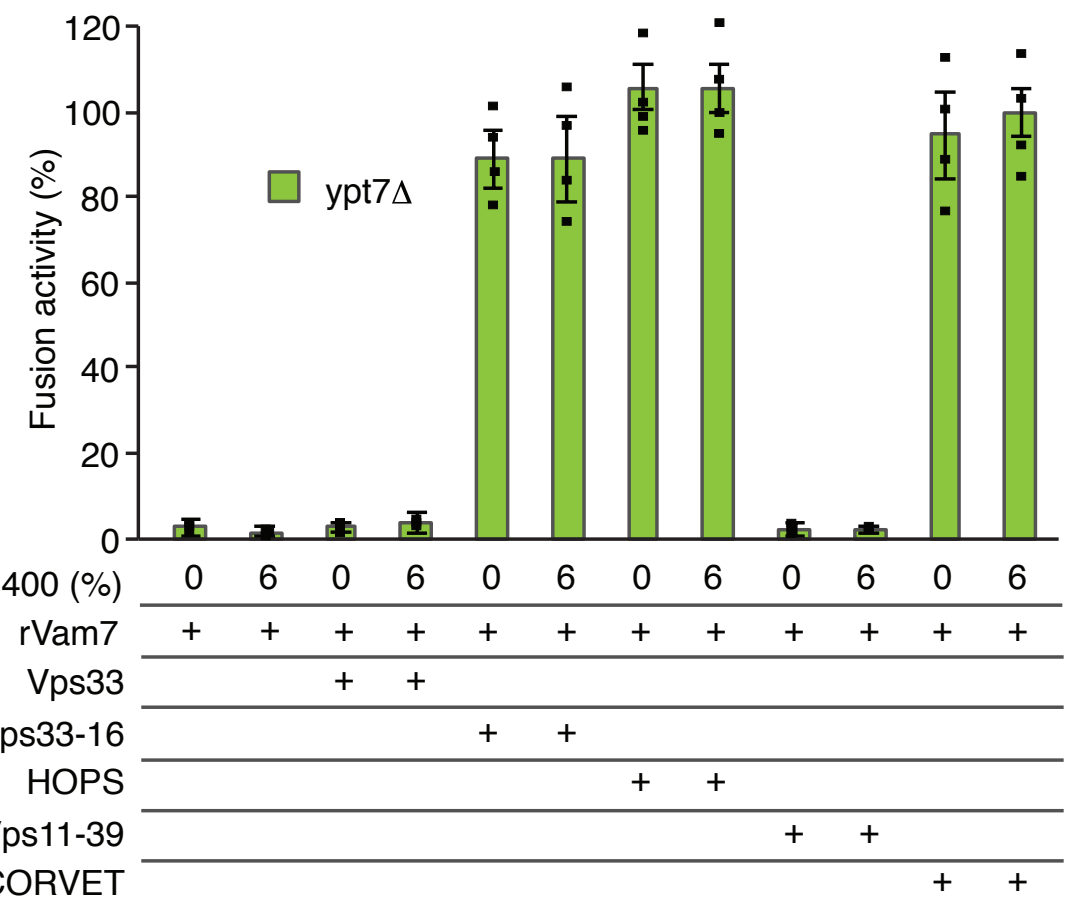




\section{Extended Data Figure 4}
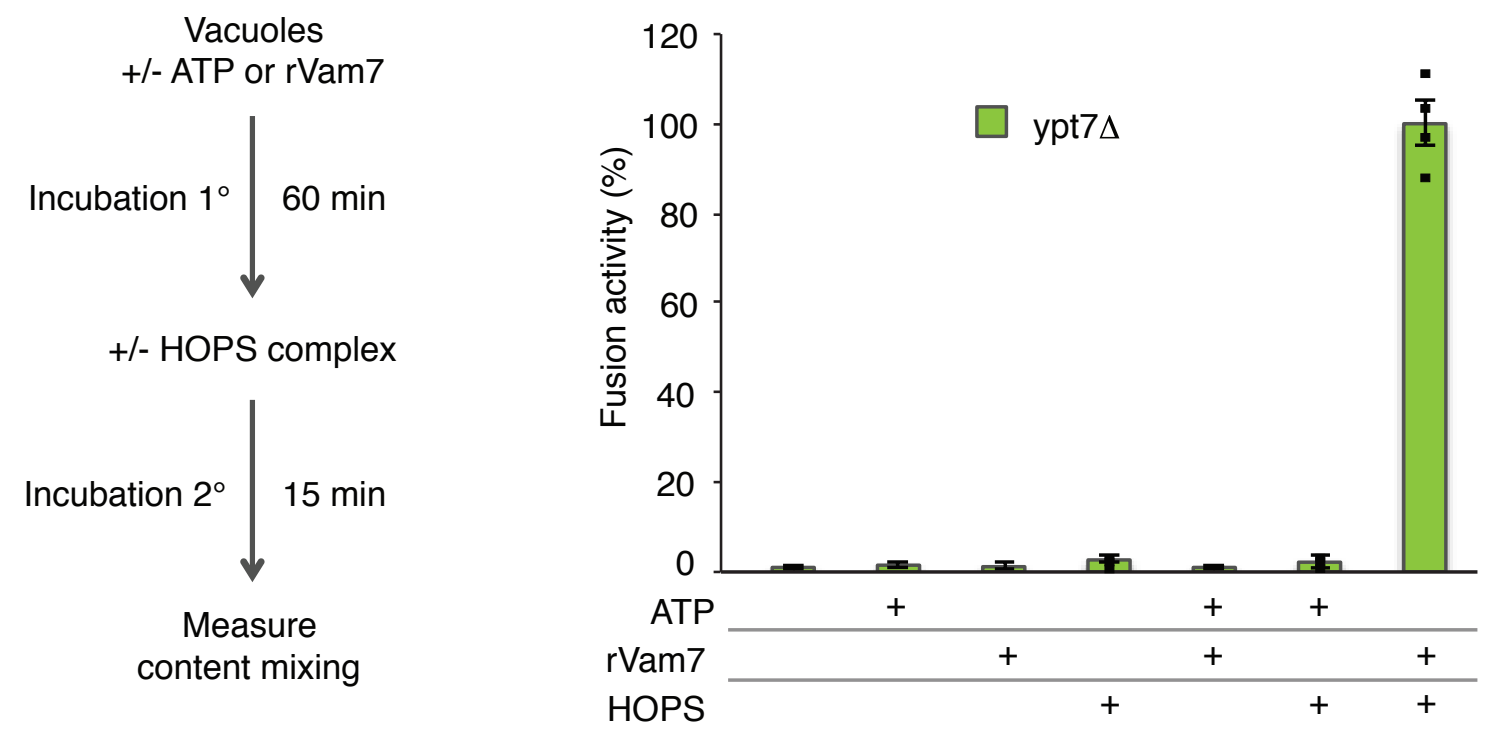


\section{Extended Data Figure 5}
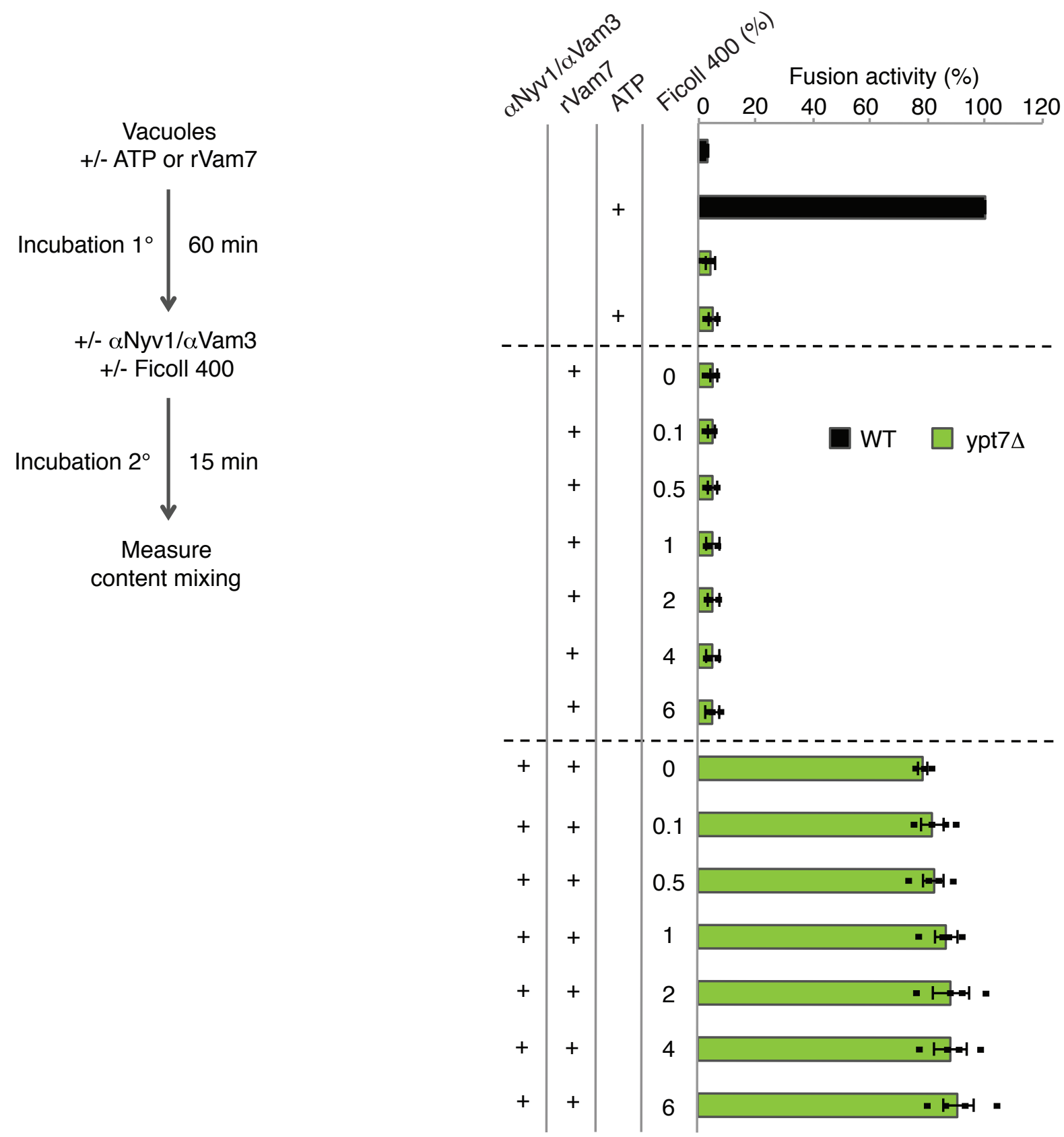


\section{Extended Data Figure 6}

a

Vam7

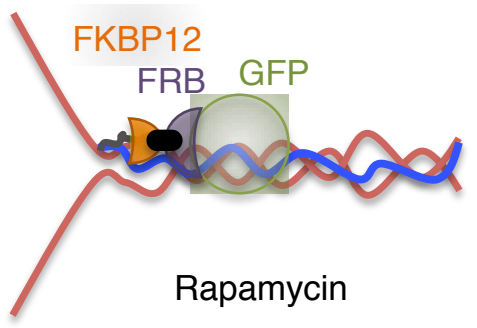

b FRB-GFP Vam7-2xFKBP12

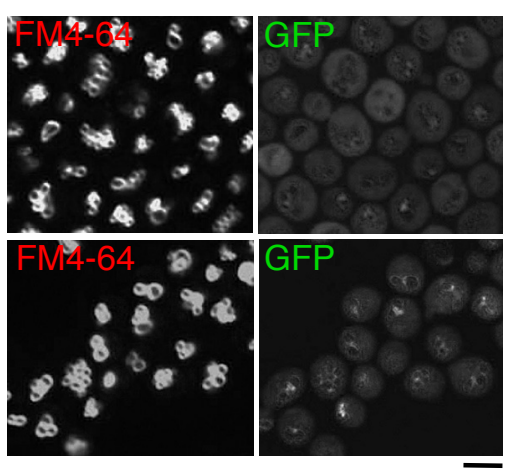

Rapamycin 


\section{Extended Data Figure 7}
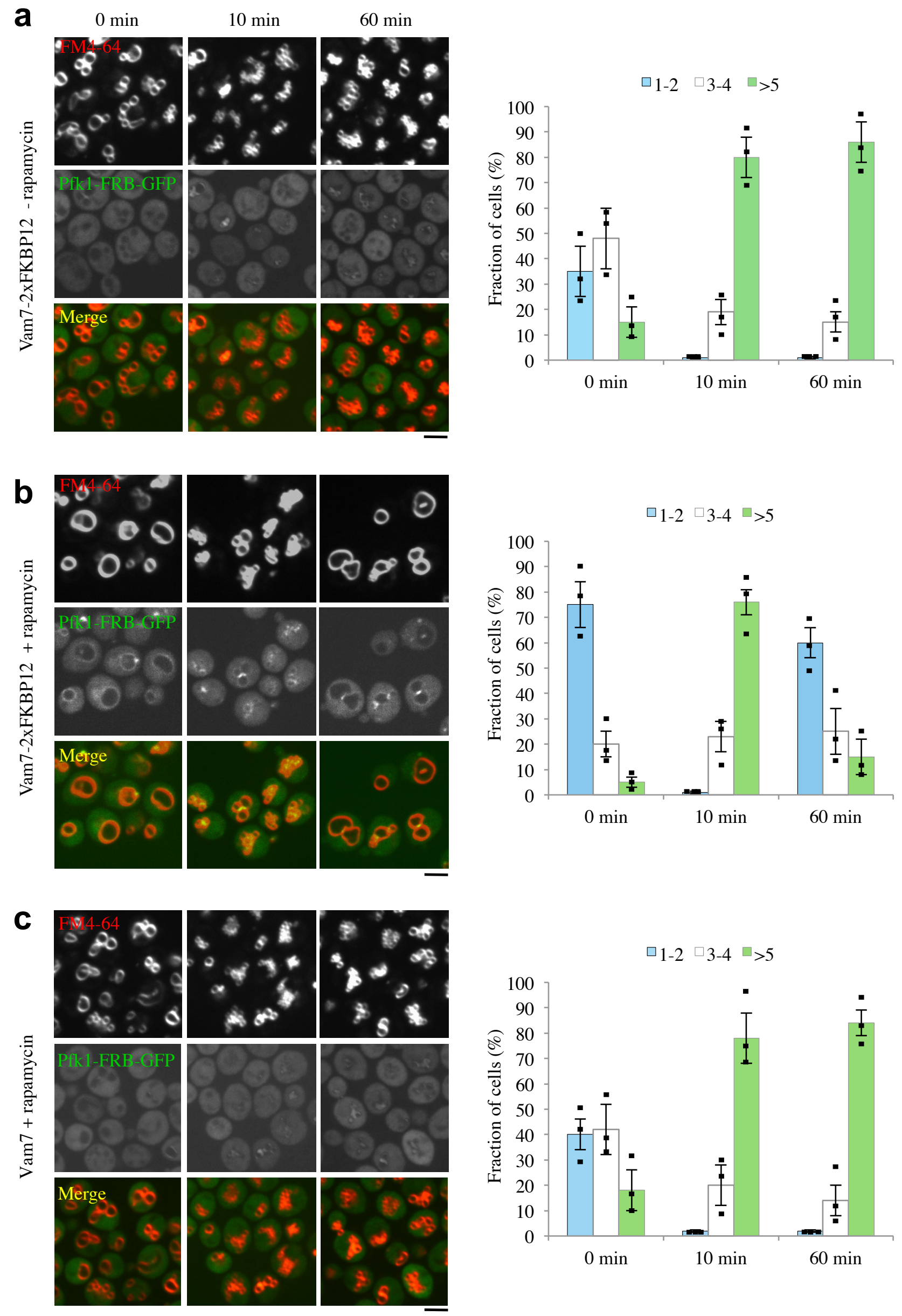


\section{Extended Data Figure 8}

a

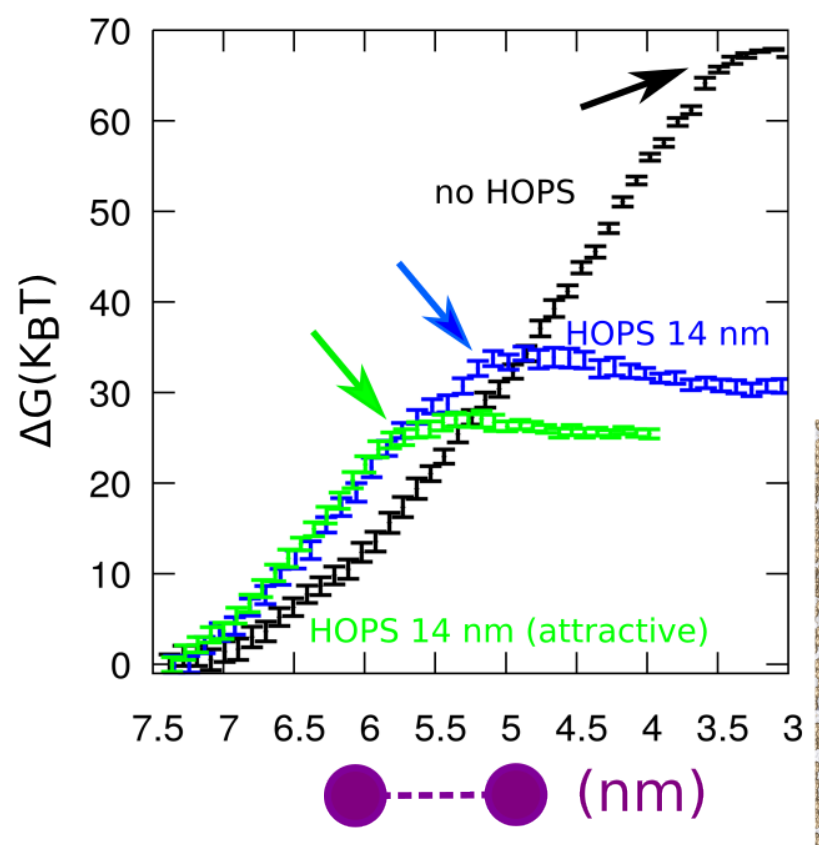

whole setup:

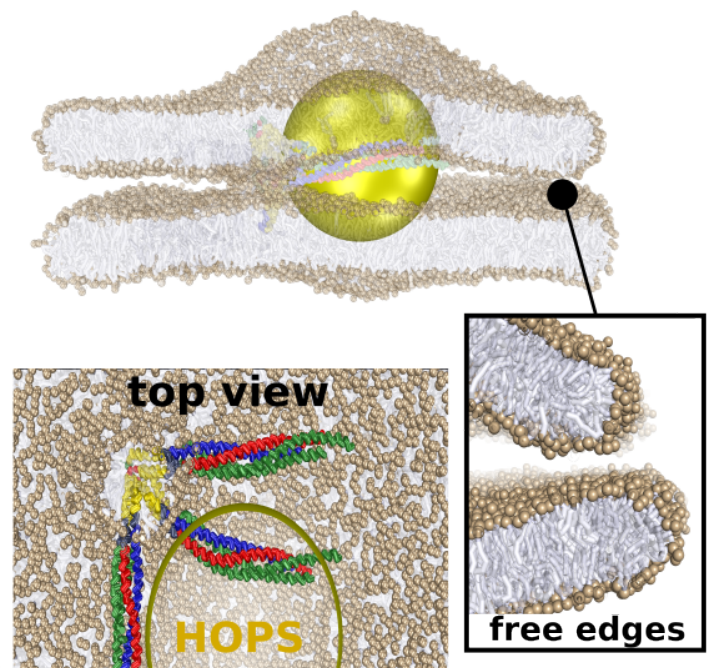

b no HOPS (40\% PE)
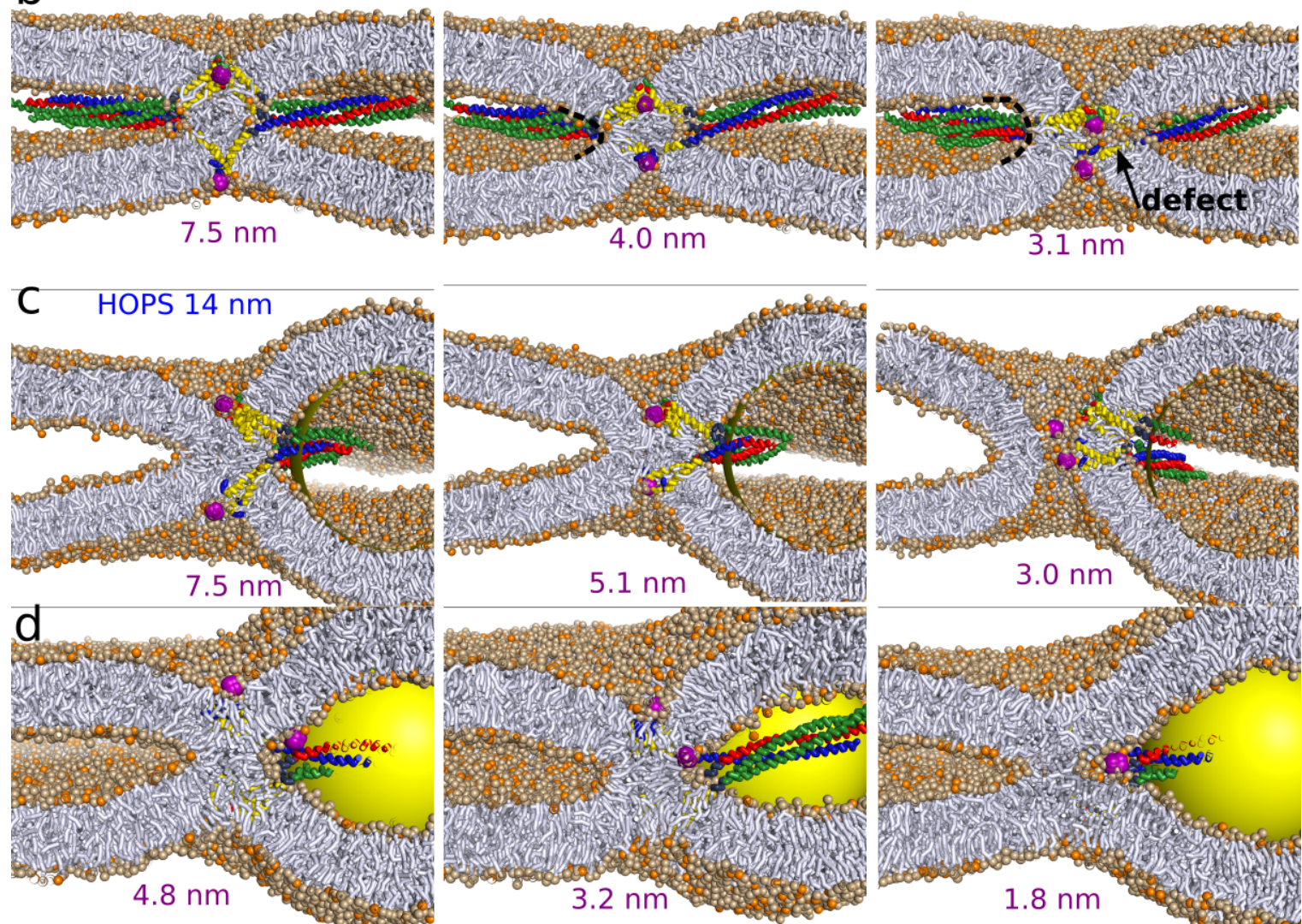


\section{Supplementary Information}

\section{Table of Contents}

Supplementary Discussion: How do SNARE ligands enhance opening of the fusion pore?.. 2

Membrane curvature as a driving force ........................................................................................ 2

The energy stored in the imposed membrane curvature .................................................... 4

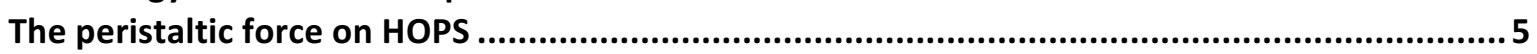

Influence of HOPS on the pulling force of SNARE transmembrane domains ..............................5

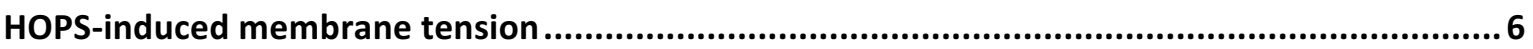

Effect on the free energy barrier of the fusion pore .................................................................. 7

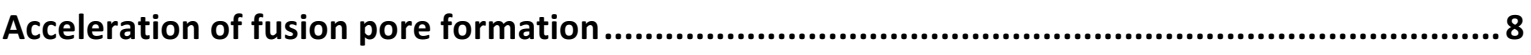

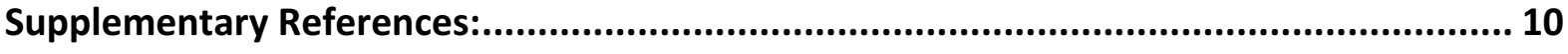

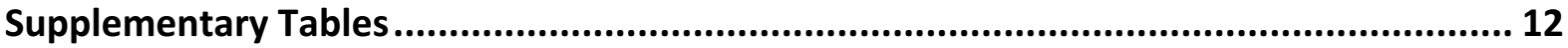

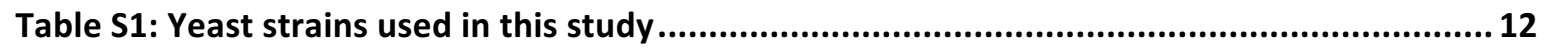

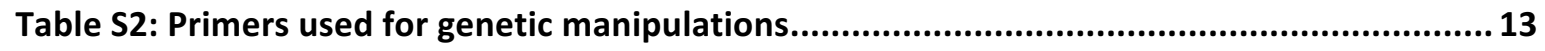

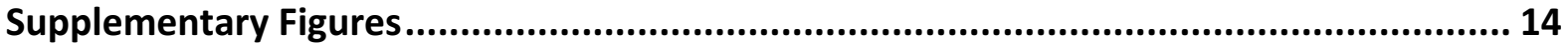

Figure S1: Full scans of blots shown in the manuscript............................................................ 14 


\section{Supplementary Discussion: How do SNARE ligands enhance opening of the fusion pore?}

\section{Membrane curvature as a driving force}

HOPS appears to contribute two separable functions to vacuole fusion. It catalyzes SNARE complex assembly ${ }^{1-4}$, which is necessary for membrane docking and the induction of hemifusion. However, it also binds to the assembled SNARE complex ${ }^{5}$ and, as we show here, this interaction promotes the formation or expansion of the fusion pore. This second function can explain why Vps33 with amino acid substitutions can support normal trans-SNARE pairing and lipid mixing but not content mixing ${ }^{6}$. It is also compatible with subsequent studies on liposome fusion, which found that the addition of the Vps33-containing HOPS complex not only increased SNARE complex formation several-fold, but also led to a disproportionately higher increase in content mixing ${ }^{7}$. While there is a priori no reason to assume that SNARE complex density should be linearly related to the rate of content mixing, this result is consistent with the steric effects of HOPS on the fusion site that we describe here.

Another unexplained finding is that SNARE ligands such as Sec17/ $\alpha$-SNAP and Sec18/NSF, which are normally involved in SNARE complex disassembly, can stimulate liposome fusion under certain conditions ${ }^{8}$. They are particularly effective when used in conjunction with a non-hydrolysable ATP-analog, which stabilizes SNARE/ $\alpha-S N A P / N S F$ complexes ${ }^{9}$. Also the fusion of intact vacuoles can be stimulated by Sec17/ $\alpha$-SNAP, but only if complete SNARE complex zippering is prevented by a C-terminal truncation of the $Q_{\mathrm{c}}$-SNARE Vam7 ${ }^{10}$. On vacuoles with wildtype SNAREs, release of Sec17 from the membrane is necessary for fusion ${ }^{11}$ and addition of Sec17 is even inhibitory ${ }^{12}$. It has been argued that binding of Sec17 might stimulate fusion by stabilizing or ordering incompletely zippered SNAREs ${ }^{10}$. This raises the question whether binding of HOPS or of SNARE antibodies might promote fusion by favoring the transition from partially zippered to fully zippered SNARE complexes. We consider this as unlikely for several reasons. First, full zippering of SNAREs is necessary to efficiently reach lipid mixing ${ }^{10}$. Since vacuoles reach a hemifused state in our experiments, we can expect them to have zippered SNARE complexes. Second, HOPS binds SNARE complexes through Vps33 and in our experiments Vps33 alone acts as a competitive inhibitor of fusion (Figs. 1,2). But 
when the effective size of Vps33 is increased by addition of monoclonal antibodies to a C-terminal peptide tag of this protein, fusion is strongly stimulated. Since this monoclonal antibody does not recognize the SNAREs, it is difficult to conceive how it might have a structuring or ordering effect on partially zippered SNARE complexes. Third, in vivo vacuole fusion can be triggered by recruiting the bulky phosphofructokinase to the SNARE complex, which is completely unrelated to fusion and unlikely to undergo any direct interaction with SNAREs. Thus, stimulation by a simple increase in SNARE complex volume remains the most suitable interpretation. By analogy, we propose that also Sec17/ $\alpha$-SNAP and Sec18/NSF, of which several units can bind the SNARE complex at a time ${ }^{13}$, stimulate liposome fusion by acting as bulky ligands that deform the fusion site. This model is also consistent with the observation that the membrane-intercalating sequences of Sec17/ $\alpha$-SNAP are dispensable for fusion as long as full-length, wildtype SNAREs are used ${ }^{9}$.

Since we can stimulate the transition from hemifusion to full fusion by bulky SNARE ligands, the SNARE domains must be sterically accessible in these intermediates. We posit that the hemifusion zone behaves like a Brownian ratchet. Spontaneous shape fluctuations of the apposed membranes might liberate the space for bulky SNARE ligands, which, once bound, fix the system in this spontaneously created, highly curved or "tense" state. Thereby, they will increase the probability of pore opening or expansion relative to the "relaxed" state, in which fluctuations into the highly curved state would be short-lived and the probability for pore formation would remain low.

Electron microscopy structures of HOPS feature a SNARE-binding domain (head region) of approximately 12-14 $\mathrm{nm}$ diameter, which probably encapsulates the SNARE complex ${ }^{14,15}$. Binding this head region to SNAREs will markedly affect the geometry of the hemifusion stalk, which restrains the apposed membranes from separating and thereby enforces strong local membrane curvature. On first sight, it may seem trivial to relate the HOPS-induced high curvature to enhanced fusion activity. Inducing curvature-stress is a well-accepted mechanism to accelerate fusion ${ }^{16}$ and small vesicles are more 'fusogenic' than large ones ${ }^{17}$. However, fusion does not relax the curvature-stress that HOPS imposes on the two flat membranes in an immediately apparent way (Fig. 4a \& Extended Data Fig. 8) and other factors, such as peristaltic forces and increased pulling on the SNARE transmembrane domains 
might play major roles. The driving force that HOPS contributes to fusion pore opening is thus not obvious - necessitating theory and simulations in order to explore its sources.

\section{The energy stored in the imposed membrane curvature}

To gain insights into the bending energy (stress) imposed on the fusion site by the HOPS-SNARE complex, we performed both molecular dynamics (MD) simulations and elastic continuum modeling (see Methods). Although a recent study reported the existence of extended hemifusion diaphragms between yeast vacuoles fusing in vitro ${ }^{18}$, our own studies by light and electron microscopy did not reveal extended hemifusion zones at a sufficient frequency to permit their quantification and interpretation (D'Agostino and Mayer, in preparation). Therefore, our models assume a stalk as the hemifused structure. Our conclusions would not qualitatively change if the hemifused structure were a diaphragm. In the MD simulations, we derived the work that HOPS must perform to bend the membrane and counteract the (partial) pressure that the membrane exerts on the surface of HOPS. In mechanical equilibrium, the bending work, which HOPS must perform to conserve the membrane shape of minimal free energy, equals the exerted pressure $(P)$ times the volume $(V)$ of HOPS. For a purely repulsive sphere this will approximate the bending energy of the adopted shape. The elastic continuum model underestimates the actual bending work by about a factor of two (see Methods for a detailed explanation). This bending energy (equilibrium bending work) amounts to about $75 \mathrm{k}_{B} T$ (12 nm HOPS sphere) to $115 \mathrm{k}_{\mathrm{B}} \mathrm{T}$ (14 nm HOPS sphere) (Extended Data Fig. 9b). These values are, however, subject to the approximation of shape, the location with respect to the stalk (e.g. Extended Data Fig. 9c), and the location within the vacuole-vacuole contact zone (at the curved periphery of the contact zone, called the vertex ring ${ }^{19}$, these values will likely be lower). Since HOPS catalyzes SNARE complex assembly, we expect that a substantial fraction of the bending work will be overcome by SNARE complex formation ( $65 \mathrm{k}_{\mathrm{B}} \mathrm{T}$ per SNARE complex $\left.{ }^{20}\right)$. Furthermore, HOPS tethers membranes through Rab-GTPases and direct lipid interaction ${ }^{21,22}$. To discern whether the acceleration of fusion might rely on the absence or presence of tether activity, we additionally modeled a HOPS with tether activity (coined ' $14 \mathrm{~nm}$ HOPS attractive') by including weak attractions between the surface of HOPS and the membrane. These surface attractions can render the average performed bending work negative (Figure S9b; green line). This implies that the bending energy is fully compensated by the 
favorable surface attraction and that the presence of HOPS will spontaneously curve the membrane also in the absence of the stalk. However, these attractions do not imply that the bending energy and thus the stress imposed on the adherent membranes vanishes (see $F_{\text {bending }}$ in Extended Data Fig. 9b,d).

\section{The peristaltic force on HOPS}

To gain insight into the force required to restrain HOPS at the fusion site, we estimated the peristaltic force $f_{d}$, which pushes HOPS away from a stalk, a fusion pore, or a SNARE complex. It originates from the induced membrane curvature and may be enhanced by osmotic pressure of the fusing vesicles. Both our MD simulations and the elastic continuum model estimate the curvature-induced component of this force to be several 10 s of $\mathrm{pN}$, up to $60 \mathrm{pN}$, when HOPS binds close to the fusion site (Extended Data Fig. 9b,d). Intuitively, we expect $f_{d}$ to decrease when HOPS is located at the vertex, i.e. at the already curved periphery of the vacuole-vacuole contact zone. However, while surface attractions (membrane tethering) reduce $f_{d}$ by half in the MD simulations (Extended Data Fig. 9b), they do not give rise to a free energy minimum near the constraint, as shown by our continuum model (Extended Data Fig. 9d). Therefore, HOPS can only be kept near the constraint by binding to the SNARE complex, which counteracts $f_{d}$.

\section{Influence of HOPS on the pulling force of SNARE transmembrane domains}

Vice versa, HOPS will exert a pulling force on the SNARE C-termini, which are thought to approach each other during the progression from hemifusion to pore opening until they closely associate as observed in the neuronal SNARE complex ${ }^{23}$. To estimate to which degree bulky SNARE complex ligands, such as HOPS, may alter the force that SNARE TMDs exert on the luminal leaflets (Extended Data Fig. 10a), we performed coarse-grained molecular dynamics simulations. We rationalized the gain in force from the reduction in relative work $(\Delta \Delta G)$ (Extended Data Fig. 10b) required to slightly bring the luminal C-termini of Vam3 and Nyv1 into closer proximity. The corresponding forces on the SNARE TMDs were derived from this relative work (Extended Data Fig. 10c). With slightly we emphasize that the hereimposed stalk indentation is reversible, meaning that the stalk will recover upon removal of the external force (no fusion barrier is being crossed). 
HOPS reduces the relative work that the SNARE complex must perform to (slightly) indent the stalk by up to $12 \mathrm{k}_{\mathrm{B}} \mathrm{T}$ (Extended Data Fig. 10b). The corresponding apparent 'force gain' is given by the derivatives of this work function (Extended Data Fig. 10c). Its magnitude of $10-30 \mathrm{pN}$ suggests that HOPS binding would gain the equivalent of one SNARE complex ${ }^{20,24,25}$. Its magnitude shows a high offset value which gradually converges as the C-termini approach each other. In contrast, simulations where the juxta-membrane regions between the coiled-coil and transmembrane domains were modeled as fully flexible (unstructured) - rendering the SNARE complex mechanically ineffective for transmitting bending force to its TMDs - showed a lower but constant 'force gain' (8 pN for 14 nm "HOPS", Fig S10c). This illustrates that the initial apparent force gain largely depends on the mechanical stiffness of the SNARE linkers. It likely originates from a projection of the peristaltic force on the SNARE C-termini. This is not necessarily intuitive since the stalk imposes an inter-membrane constraint and therefore one might expect that the imposed stress tends to stretch the stalk rather than ease its indentation.

The observed force gain is compromised by positioning "HOPS" more distally with respect to the stalk (Extended Data Fig. 10b,c; HOPS $12 \mathrm{~nm}$ (linker)). This suggests that the force gain is mediated by direct steric effects of HOPS on the site of hemifusion. Although the force gain is substantial with respect to the inherent force exerted by a SNARE complex - doubling or even tripling the effective SNARE force -- the concomitant gain in free energy, i.e., the driving force of fusion, remains (I) relatively small $\left(\sim 10 \mathrm{k}_{\mathrm{B}} \mathrm{T}\right)$ and $(\mathrm{II})$ largely relies on the mechanical stiffness and thus the adopted secondary structure of the HOPS-bound SNAREs.

\section{HOPS-induced membrane tension}

The curvature induced by HOPS reduces the effective, projected area of the proximal leaflets via corrugation of the membrane surface. This may result in a concomitant surface tension. It is unclear whether such a (local) tension could relax (dissipate) via lipid diffusion, solvent efflux and/or lipid flip-flops (in case of asymmetric leaflet tension or spontaneous curvature). We used our continuum model to estimate the membrane area $\left(A-A_{0}\right)$ that would be required to compensate for this tension induced by the presence of HOPS (Extended Data Fig. 9c). A single HOPS sphere, which is closely restrained to the fusion site, will reduce the area of the membrane-membrane contact zone by $\sim 70 \mathrm{~nm}^{2}$. Although this may in principle 
generate significant tension at high densities of HOPS on the membrane, we can effectively rule out tension as a main cause of accelerated fusion due to our experimental observations: Displacing bulky SNARE ligands from the SNARE complex via a flexible linker of 35 amino acids abolishes the stimulation of fusion by them (Fig. 3). But the required excess membrane area $\left(A-A_{0}\right)$, and thereby the tension that HOPS might induce, depends only weakly on the distance of HOPS (d) from the inter-membrane constraint - quite in contrast to $F_{\text {bending }}$ and $f_{d}$ (Figure S9c,d). Therefore, tension should not provide the main driving force for the observed acceleration of fusion.

\section{Effect on the free energy barrier of the fusion pore}

We explored the effect of 'HOPS' on the free energy barrier of fusion pore formation using a previously published method ${ }^{26}$. In order to estimate the free energy required to open the fusion pore, we pull two hydrophilic 'beads' (probes; colored purple in Extended Data Fig. 8b-d) towards each other near the center of the stalk. Each probe is comprised of 8 clustered solvent beads. Bringing the probes in closer proximity exerts a squeezing force on the stalk which enforces its expansion (evolution). The "stalk thickness" is defined by the distance between the two probes. The idea of this approach is that one brings the system close to a nucleation barrier until the barrier can be crossed spontaneously within the simulation time scales. The work required to enforce nucleation gives an estimate of the height of the barrier. An advantage of this approach is that we can use the same reaction coordinate to test whether there is a propensity for 'leaky' fusion (coined leakage pore mediated stalk elongation ${ }^{26}$ ). For more detailed information see our previous work ${ }^{26}$.

The membrane system that we simulated consists of a POPC:POPC mixture $(40 \%$ POPE) with a fusion site comprised of one HOPS-bound SNARE complex and two additional unbound SNARE complexes (see Extended Data Fig. 8a and Methods). Tension-less membrane conditions are ensured by the presence of free membrane edges which allow fast spontaneous lipid flip-flops between the leaflets and free adaptation of membrane area. The presence of the HOPS sphere substantially reduces the free energy barrier of fusion pore formation, from $67 k_{B} T$ to $34 k_{B} T$ (Extended Data Fig. 8a). A striking barrier of $67 \mathrm{k}_{\mathrm{B}} \mathrm{T}$ - despite the presence of three SNARE complexes - illustrates how 'trapped' the fusion reaction is after stalk formation. Furthermore, metastable hemifusion diaphragms, i.e., hemifusion states 
of the thickness of a single membrane, have not been observed in the simulations, suggesting that the stalk to fusion pore transition faces only a single free energy barrier. Since HOPS is assumed to tether membranes in vivo ${ }^{21,22,27}$, it is important to test whether the observed 'fusion acceleration' is conserved (if not enhanced) even in the presence of membrane tethering, which we have approximated by making HOPS membrane-attractive (no net bending work; Extended Data Fig. 9b). This is the case. The pronounced reduction of work required to open the fusion pore can thus be attributed to a substantial shift in the nucleation barrier towards larger probe to probe distances (the arrows in Extended Data Fig. 8a). In other words, opening of the fusion pore requires way less squeezing of the stalk - and thus less work - in the presence HOPS. This results in a more than $30 \mathrm{k}_{\mathrm{B}} \mathrm{T}$ decrease of the fusion barrier and thereby a dramatic acceleration $\left(>\mathrm{e}^{30}\right)$ of the subsequent fusion reaction.

\section{Acceleration of fusion pore formation}

Based on the preceding considerations, we propose two possible sources for the observed acceleration of fusion pore formation by HOPS:

(I) Relaxation of curvature stress. The progression of SNARE zippering into the juxta-membrane and transmembrane domains upon fusion pore opening moves HOPS further away from the fusion site - it increases the distance d (Extended Data Fig. 9) by about $1 \mathrm{~nm}$. HOPS thereby imposes less of a steric constraint. The continuum model illustrates that the bending energy $\left(F_{\text {bending }}\right)$ features a sharp, initial reduction when a nearby located HOPS complex moves slightly away from the stalk. From the MD simulations, we estimate that fusion pore formation reduces the bending work that HOPS performs by about $30 \mathrm{k}_{\mathrm{B}} \mathrm{T}$. This partial, relative release of bending stress may very well drive subsequent fusion pore opening.

(II) Geometrical compatibility. Fusion pore formation involves the formation of pronounced 'wings' resulting in a teardrop shape ${ }^{28,29}$ in order to reduce the curvature of the pore interface. A stalk opposes this rearrangement because it forces the trans-leaflets to remain largely parallel/horizontal ${ }^{30}$. The dashed lines in Figure S8b illustrate a fast decrease in curvature upon initial fusion pore opening. Here, the pre-existing curvature induced by HOPS (essentially a teardrop shape) is geometrically more compatible with a fusion pore than a stalk. This provides a relative free energy advantage for fusion pore formation. 
In the absence of HOPS, the local curvature associated with nucleation of a fusion pore in flat membranes is so high that it seemingly becomes advantageous to form membrane defects near the transmembrane domains of the SNAREs (Extended Data Fig. 8b). Such 'leaky' transitions are not observed in the presence of HOPS, probably because it lowers the threshold for fusion pore nucleation and thereby channels the reaction towards non-leaky fusion. We have also tested directly whether HOPS might render the fusion site prone to membrane rupture. To this end we placed the two probes such that pulling them together would locally disrupt the membrane near the stalk (see Extended Data Fig. 8d). Enforcing such a membrane defect does not result in membrane rupture but the defect self-heals. 


\section{Supplementary References:}

1. Sato, T. K., Rehling, P., Peterson, M. R. \& Emr, S. D. Class C Vps protein complex regulates vacuolar SNARE pairing and is required for vesicle docking/fusion. Mol Cell 6, 661-671 (2000).

2. Orr, A., Song, H., Rusin, S. F., Kettenbach, A. N. \& Wickner, W. HOPS catalyzes the interdependent assembly of each vacuolar SNARE into a SNARE complex. Mol. Biol. Cell 28, 975-983 (2017).

3. Krämer, L. \& Ungermann, C. HOPS drives vacuole fusion by binding the vacuolar SNARE complex and the Vam7 PX domain via two distinct sites. Mol. Biol. Cell 22, 2601-2611 (2011).

4. Baker, R. W. et al. A direct role for the Sec1/Munc18-family protein Vps33 as a template for SNARE assembly. Science 349, 1111-1114 (2015).

5. Lobingier, B. T. \& Merz, A. J. Sec1/Munc18 protein Vps33 binds to SNARE domains and the quaternary SNARE complex. Mol. Biol. Cell 23, 4611-4622 (2012).

6. Pieren, M., Schmidt, A. \& Mayer, A. The SM protein Vps33 and the t-SNARE $\mathrm{H}(\mathrm{abc})$ domain promote fusion pore opening. Nat Struct Mol Biol 17, 710-717 (2010).

7. Zick, M. \& Wickner, W. T. A distinct tethering step is vital for vacuole membrane fusion. elife 3, e03251 (2014).

8. Zick, M., Orr, A., Schwartz, M. L., Merz, A. J. \& Wickner, W. T. Sec17 can trigger fusion of trans-SNARE paired membranes without Sec18. PNAS 112, E2290-E2297 (2015).

9. Song, H., Orr, A., Duan, M., Merz, A. J. \& Wickner, W. Sec17/Sec18 act twice, enhancing membrane fusion and then disassembling cis-SNARE complexes. elife 6, 1111 (2017).

10. Schwartz, M. L. \& Merz, A. J. Capture and release of partially zipped transSNARE complexes on intact organelles. J Cell Biol 185, 535-549 (2009).

11. Mayer, A., Wickner, W. \& Haas, A. Sec18p (NSF)-driven release of Sec17p (alpha-SNAP) can precede docking and fusion of yeast vacuoles. Cell 85, 8394 (1996).

12. Wang, L., Ungermann, C. \& Wickner, W. The docking of primed vacuoles can be reversibly arrested by excess Sec17p (alpha-SNAP). 275, 22862-22867 (2000).

13. Zhao, M. et al. Mechanistic insights into the recycling machine of the SNARE complex. Nature (2015). doi:10.1038/nature14148

14. Kuhlee, A., Raunser, S. \& Ungermann, C. Functional homologies in vesicle tethering. FEBS Lett 589, 2487-2497 (2015).

15. Bröcker, C. et al. Molecular architecture of the multisubunit homotypic fusion and vacuole protein sorting (HOPS) tethering complex. PNAS 109, 1991-1996 (2012).

16. Martens, S., Kozlov, M. M. \& McMahon, H. T. How synaptotagmin promotes membrane fusion. Science 316, 1205-1208 (2007).

17. Hernandez, J. M., Kreutzberger, A. J. B., Kiessling, V., Tamm, L. K. \& Jahn, R. Variable cooperativity in SNARE-mediated membrane fusion. PNAS 111, 12037-12042 (2014).

18. Mattie, S., McNally, E. K., Karim, M. A., Vali, H. \& Brett, C. L. How and why intralumenal membrane fragments form during vacuolar lysosome fusion. Mol. Biol. Cell 28, 309-321 (2017).

19. Wang, L., Merz, A. J., Collins, K. M. \& Wickner, W. Hierarchy of protein assembly at the vertex ring domain for yeast vacuole docking and fusion. $J$ 
Cell Biol 160, 365-374 (2003).

20. Gao, Y. et al. Single reconstituted neuronal SNARE complexes zipper in three distinct stages. Science 337, 1340-1343 (2012).

21. Orr, A., Wickner, W., Rusin, S. F., Kettenbach, A. N. \& Zick, M. Yeast vacuolar HOPS, regulated by its kinase, exploits affinities for acidic lipids and Rab:GTP for membrane binding and to catalyze tethering and fusion. Mol. Biol. Cell 26, 305-315 (2015).

22. Lürick, A. et al. Multivalent Rab interactions determine tether-mediated membrane fusion. Mol. Biol. Cell mbc.E16-11-0764 (2016).

doi:10.1091/mbc.E16-11-0764

23. Stein, A., Weber, G., Wahl, M. C. \& Jahn, R. Helical extension of the neuronal SNARE complex into the membrane. Nature 460, 525-528 (2009).

24. Zorman, S. et al. Common intermediates and kinetics, but different energetics, in the assembly of SNARE proteins. elife 3, e03348-e03348 (2013).

25. Zhang, X. et al. Stability, folding dynamics, and long-range conformational transition of the synaptic t-SNARE complex. Proc. Natl. Acad. Sci. U.S.A. 113, E8031-E8040 (2016).

26. Risselada, H. J., Bubnis, G. \& Grubmüller, H. Expansion of the fusion stalk and its implication for biological membrane fusion. 111, 11043-11048 (2014).

27. Hickey, C. M. \& Wickner, W. HOPS initiates vacuole docking by tethering membranes before trans-SNARE complex assembly. Mol. Biol. Cell 21, 22972305 (2010).

28. Ryham, R. J., Ward, M. A. \& Cohen, F. S. Teardrop shapes minimize bending energy of fusion pores connecting planar bilayers. Phys. Rev. E 88, 693 (2013).

29. Jackson, M. B. Minimum Membrane Bending Energies of Fusion Pores. $J$ Membr Biol 231, 101-115 (2009).

30. Kozlovsky, Y., Efrat, A., Siegel, D. P., Siegel, D. A. \& Kozlov, M. M. Stalk phase formation: effects of dehydration and saddle splay modulus. Biophys $J$ 87, 2508-2521 (2004).

31. Jones, E. W., Zubenko, G. S. \& Parker, R. R. PEP4 gene function is required for expression of several vacuolar hydrolases in Saccharomyces cerevisiae. Genetics 102, 665-677 (1982).

32. Haas, A., Conradt, B. \& Wickner, W. G-protein ligands inhibit in vitro reactions of vacuole inheritance. J Cell Biol 126, 87-97 (1994).

33. Ostrowicz, C. W. et al. Defined subunit arrangement and rab interactions are required for functionality of the HOPS tethering complex. Traffic 11, 13341346 (2010). 
Supplementary Tables

Table S1: Yeast strains used in this study

\begin{tabular}{|c|c|c|}
\hline Strain & Genotype & Reference \\
\hline BJ3505 & $\begin{array}{l}\text { MATa pep4::HIS3 prb1- } \Delta \text { 1.6R lys2-208 trp1- } \Delta \\
101 \text { ura3-52 gal2 can }\end{array}$ & 31 \\
\hline DKY6281 & $\begin{array}{l}\text { MATa pho8::TRP1 leu2-3 leu2-112 lys2-801 } \\
\text { suc2- } \Delta 9 \operatorname{trp1-~} \Delta 901 \text { ura3-52 }\end{array}$ & 32 \\
\hline BY4742 & MATa his3-1 leu2-0 met15-0 ura3-0 & Lab stock \\
\hline BJ Vam3-myc & BJ3505; Vam3-His6(myc)2::URA & \\
\hline BJ Nyv1-HA & BJ3505; Nyv1-His6(HA)3::URA & 6 \\
\hline BJ ypt7 $\Delta$ & BJ3505; ypt7::G418 & Lab stock \\
\hline DKY ypt7 $\Delta$ & DKY6281; ypt7::G418 & Lab stock \\
\hline BJ ypt7 $\Delta$ Nyv1-HA & BJ3505 ypt7 $\Delta ;$ Nyv1-His6(HA)3::URA & This study \\
\hline BJ ypt7 $\Delta$ Vam3-myc & BJ3505 ypt7 $\Delta$; Vam3-His6(myc)2::URA & This study \\
\hline $\begin{array}{l}\text { BY tor1-1 Vam7- } \\
\text { 2xFKBP12 }\end{array}$ & BY4742; Vam7-2xFKBP12::URA & This study \\
\hline $\begin{array}{l}\text { BY tor1-1 Vam7- } \\
\text { 2xFKBP12 Pfk1- } \\
\text { FRB-GFP }\end{array}$ & $\begin{array}{l}\text { BY4742; Vam7-2xFKBP12::URA ; Pfk1-FRB- } \\
\text { GFP::G418 }\end{array}$ & This study \\
\hline $\begin{array}{l}\text { BY tor1-1 Pfk1- } \\
\text { FRB-GFP }\end{array}$ & BY4742; Pfk1-FRB-GFP::G418 & This study \\
\hline $\begin{array}{l}\text { BY tor1-1 Vam7-LL- } \\
\text { 2xFKBP12 }\end{array}$ & BY4742; Vam7-LL-2xFKBP12::URA & This study \\
\hline $\begin{array}{l}\text { BY tor1-1 Vam7-LL- } \\
\text { 2xFKBP12 }\end{array}$ & & \\
\hline Pfk1-FRB-GFP & $\begin{array}{l}\text { BY4742; Vam7-LL-2xFKBP12::URA ; Pfk1- } \\
\text { FRB-GFP::G418 }\end{array}$ & This study \\
\hline $\begin{array}{l}\text { BY tor1-1 Vam7- } \\
\text { 2xFKBP12 + FRB- } \\
\text { GFP }\end{array}$ & $\begin{array}{l}\text { BY4742; Vam7-2xFKBP12::URA ; pRS415- } \\
\text { TEFpr-FRB-GFP (LEU) }\end{array}$ & This study \\
\hline $\begin{array}{l}\text { CUY2675 GAL- } \\
\text { HOPS Vps41-TAP }\end{array}$ & $\begin{array}{l}\text { MATa/alpha his3 } \Delta 200 \text { leu2 } \Delta 0 / \text { leu2 } \Delta 0 \text { lys } 2 \Delta 0 \\
\text { met15 } \Delta 0 / \text { met15 } \Delta 0 \text { trp1 } 1 \Delta 63 / \operatorname{trp} 1 \Delta 63 \\
\text { ura3 } \Delta 0 / \text { ura3 } \Delta 0 \text { VPS11pr::HIS3-GAL1pr } \\
\text { VPS16::natNT2-GAL1pr VPS18::KanMX- } \\
\text { GAL1pr-3HA VPS33::TRP1-GAL1pr } \\
\text { VPS41::TRP-GAL1pr-TAP-URA3 } \\
\text { VPS39::HIS3-GALpr }\end{array}$ & 33 \\
\hline $\begin{array}{l}\text { CUY3238 GAL- } \\
\text { Vps33-16 Vps16- } \\
\text { TAP }\end{array}$ & $\begin{array}{l}\text { MATa his3 } \Delta 200 \text { leu2 } \Delta 0 \text { lys } 2 \Delta 0 \text { met } 15 \Delta 0 \\
\operatorname{trp} 1 \Delta 63 \text { ura3 } \Delta 0 \text { VPS33::HIS3-GALpr } \\
\text { Vps16::TRP-GAL1pr-TAP-URA3 }\end{array}$ & 33 \\
\hline $\begin{array}{l}\text { CUY4307 GAL- } \\
\text { Vps39-11 Vps39- } \\
\text { TAP }\end{array}$ & $\begin{array}{l}\text { MATa/alpha his3 } \Delta 200 \text { leu2 } \Delta 0 / \text { leu2d0 lys } 2 \Delta 0 \\
\text { met15 } \Delta 0 / \text { met15d0 trp1 } 163 / \operatorname{trp} 1 \Delta 63 \\
\text { ura3 } \Delta 0 / \text { ura3 } \Delta 0 \text { VPS11pr::HIS3-GAL1pr } \\
\text { VAM6pr::KanMX-GAL1pr VAM6::TAP-URA3 }\end{array}$ & 33 \\
\hline
\end{tabular}




\begin{tabular}{|c|c|c|}
\hline $\begin{array}{l}\text { CUY4895 GAL- } \\
\text { CORVET Vps8-TAP }\end{array}$ & $\begin{array}{l}\text { MATa/alpha his3 } \Delta 200 \text { leu2 } \Delta 0 / \text { leu2 } \Delta 0 \text { lys } 2 \Delta 0 \\
\text { met15 } \Delta 0 / \text { met15 } \Delta 0 \text { trp } 1 \Delta 63 / \operatorname{trp} 1 \Delta 63 \\
\text { ura3 } \Delta 0 / \text { ura3 } \Delta 0 \text { VPS11pr::HIS3-GAL1pr } \\
\text { VPS16::natNT2-GAL1pr VPS18::KanMX- } \\
\text { GAL1pr-3HA VPS33::TRP1-GAL1pr } \\
\text { VPS8::TRP-GAL1pr-TAP-URA3 VPS3::HIS3- } \\
\text { GALpr }\end{array}$ & 33 \\
\hline $\begin{array}{l}\text { CUY8919 GAL- } \\
\text { Vps33-TAP }\end{array}$ & $\begin{array}{l}\text { MATa his } 3 \Delta 200 \text { leu2 } \Delta 0 \text { lys } 2 \Delta 0 \text { met } 15 \Delta 0 \\
\operatorname{trp} 1 \Delta 63 \text { ura3 } \Delta 0 \text { VPS33::HIS3-GALpr-TAP- } \\
\text { URA3 }\end{array}$ & 33 \\
\hline
\end{tabular}

Table S2: Primers used for genetic manipulations

\begin{tabular}{|c|c|}
\hline Primer & equence \\
\hline Fw Vam3-myc & $\begin{array}{l}\text { ATTATAATAGTTGTGTGCATGGTGGTATTGCTTGCTGTATTAAGTTCCC } \\
\text { ACCACCATCATCATCAC }\end{array}$ \\
\hline Rv Vam3-myc & $\begin{array}{l}\text { TAATCTCCTTAAACGCGCATTGAGCACAGACTTTCTGGTAGACCCACTA } \\
\text { TAGGGAGACCGGCAGATC }\end{array}$ \\
\hline Fw Nyv1-HA & $\begin{array}{l}\text { ATTATACTATTTGTAAGTGCTGCTTTCATGTTTTTCTATCTGTGGTCCCA } \\
\text { CCACCATCATCATCAC }\end{array}$ \\
\hline Rv Nyv1-HA & $\begin{array}{l}\text { GTAAATAAAAAAAAAGGGGAGCTGTCCCACGACAATAACATTAATACTA } \\
\text { TAGGGAGACCGGCAGATC }\end{array}$ \\
\hline $\begin{array}{l}\text { Fw } \\
\text { GFI }\end{array}$ & $\begin{array}{l}\text { AGAGCTGAGGTAGCCGCTTTAGCCGCTGAAAAC } \\
\text { GCTAGCATCCTCTGGC }\end{array}$ \\
\hline $\begin{array}{l}\text { Rv Pfk1-FRB- } \\
\text { GFP }\end{array}$ & $\begin{array}{l}\text { ATTTTTTACCTCCTTTTGCTTAACTTAAACTTTTTCATTGCAATCAT } \\
\text { AATTCGAGCTCG }\end{array}$ \\
\hline $\begin{array}{l}\text { Fw Vam7- } \\
\text { 2xFKBP12 }\end{array}$ & $\begin{array}{l}\text { GGAGGTTACAGATAGCCAACAAGAAGGCTAGACATTTTAACAACAGTG } \\
\text { CTGGTCGACGGATCGGTGACGGTGCTGGT }\end{array}$ \\
\hline $\begin{array}{l}\mathrm{RV} \\
2 \mathrm{RFI}\end{array}$ & $\begin{array}{l}\text { CAAATATACTCTCAGGATTTGTAACCCGGATAGTAACTCATTAAT } \\
\text { GAATTCGAGCTCG }\end{array}$ \\
\hline $\begin{array}{l}\text { Fw Vam7-LL- } \\
\text { 2xFKBP12 }\end{array}$ & $\begin{array}{l}\text { GGAGGTTACAGATAGCCAACAAGAAGGCTAGACATTTTAACAACAGTG } \\
\text { CTAGTCTAAGTGGTGGTGGTGGTTCTGGAGGAGGAGGATCCGGTGGT } \\
\text { GGAGGAAGTGGAGGTGGAGGTGCTGCCGCAGGTGACGGTGCTGGTTT } \\
\text { AATT }\end{array}$ \\
\hline & $\mathrm{CCC}$ \\
\hline Rv FRB & AGCTCTTATTTGTATAGTT \\
\hline
\end{tabular}




\section{Supplementary Figures}

Figure S1: Full scans of blots shown in the manuscript

Red boxes indicate the cropped regions. Molecular weight markers are indicated in the respective figures.

Fig. 1a

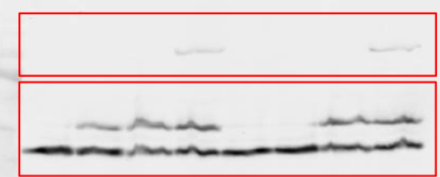

Fig. 2d

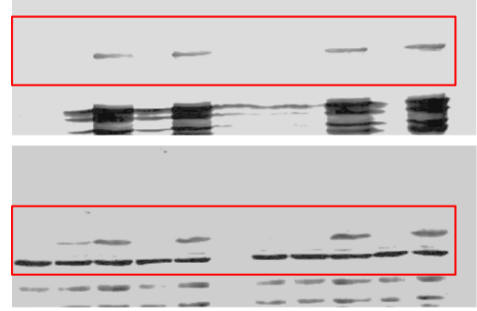

Ext. Data Fig. 1a

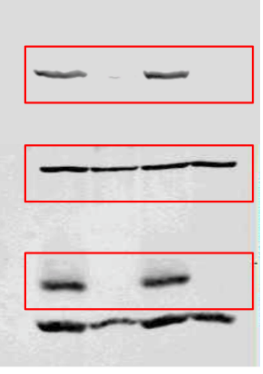

Ext. Data Fig. 1a

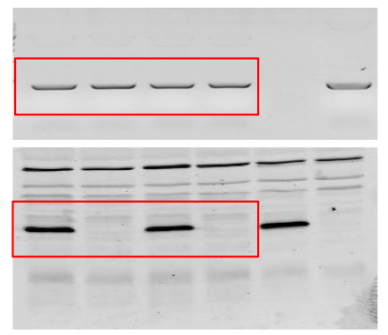

Ext. Data Fig. 1d

Ext. Data Fig. 1e

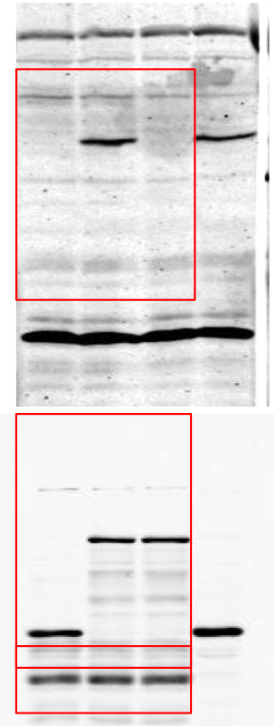

Ext. Data Fig. 2b

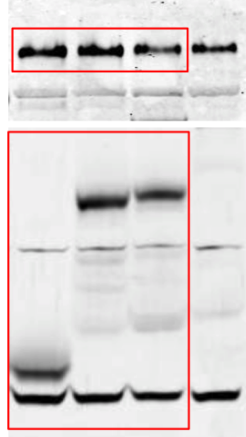

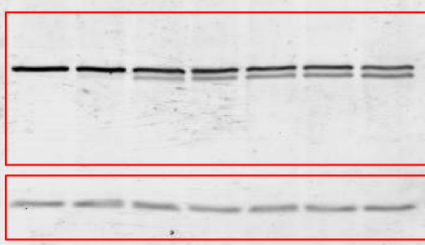

